\title{
Short-Term Facilitation at a Detonator Synapse Requires the Distinct Contribution of Multiple Types of Voltage-Gated Calcium Channels
}

\author{
Simon Chamberland, (D)Alesya Evstratova, and Katalin Tóth \\ Quebec Mental Health Institute, Department of Psychiatry and Neuroscience, Université Laval, Quebec City, Quebec, G1J 2G3 Canada
}

Neuronal calcium elevations are shaped by several key parameters, including the properties, density, and the spatial location of voltagegated calcium channels (VGCCs). These features allow presynaptic terminals to translate complex firing frequencies and tune the amount of neurotransmitter released. Although synchronous neurotransmitter release relies on both P/Q- and N-type VGCCs at hippocampal mossy fiber-CA3 synapses, the specific contribution of VGCCs to calcium dynamics, neurotransmitter release, and short-term facilitation remains unknown. Here, we used random-access two-photon calcium imaging together with electrophysiology in acute mouse hippocampal slices to dissect the roles of P/Q- and N-type VGCCs. Our results show that N-type VGCCs control glutamate release at a limited number of release sites through highly localized $\mathrm{Ca}^{2+}$ elevations and support short-term facilitation by enhancing multivesicular release. In contrast, $\mathrm{Ca}^{2+}$ entry via $\mathrm{P} / \mathrm{Q}$-type VGCCs promotes the recruitment of additional release sites through spatially homogeneous $\mathrm{Ca}^{2+}$ elevations. Altogether, our results highlight the specialized contribution of P/Q- and N-types VGCCs to neurotransmitter release.

Key words: mossy fiber; short-term plasticity; voltage-gated calcium channels

\section{Significance Statement}

In presynaptic terminals, neurotransmitter release is dynamically regulated by the transient opening of different types of voltagegated calcium channels. Hippocampal giant mossy fiber terminals display extensive short-term facilitation during repetitive activity, with a large several fold postsynaptic response increase. Though, how giant mossy fiber terminals leverage distinct types of voltage-gated calcium channels to mediate short-term facilitation remains unexplored. Here, we find that P/Q- and N-type VGCCs generate different spatial patterns of calcium elevations in giant mossy fiber terminals and support short-term facilitation through specific participation in two mechanisms. Whereas N-type VGCCs contribute only to the synchronization of multivesicular release, P/Q-type VGCCs act through microdomain signaling to recruit additional release sites.

\section{Introduction}

Presynaptic terminals are specialized structures that possess the ability to rapidly convert electrical impulses to neurotransmitter release. This is made possible by their highly localized expression of a wide arsenal of voltage-gated sodium, potassium, and $\mathrm{Ca}^{2+}$

\footnotetext{
Received Jan. 18, 2017; revised March 9, 2017; accepted March 15, 2017.

Author contributions:S.C., A.E., and K.T. designed research;S.C., A.E., and K.T. performed research;S.C., A.E., and K.T. analyzed data; S.C., A.E., and K.T. wrote the paper.

This work was supported by the Canadian Institutes of Health Research (K.T.) and the National Science and Engineering Research Council (K.T.); S.C. received PhD Fellowships from NSERC and CTRN; and A.E. received a PhD fellowship from CTRN. We thank Dr. Stéphane Dieudonné for advice with RAMP microscopy and two-photon calcium imaging, Mr. Benjamin Mathieu for technical support and software development, and Dr. Simon Labrecque for assistance with automation of calcium imaging data analysis.

The authors declare no competing financial interests.

Correspondence should be addressed to Dr. Katalin Tóth, Quebec Mental Health Institute, Department of Psychiatry and Neuroscience, Faculty of Medicine, Université Laval, 2601 chemin de la Canardière, Quebec City, QC, G1J 2 G3 Canada. E-mail: toth.katalin@crulrg.ulaval.ca.

DOI:10.1523/JNEUROSCI.0159-17.2017

Copyright $\odot 2017$ the authors $\quad 0270-6474 / 17 / 374913-15 \$ 15.00 / 0$
}

channels (Geiger and Jonas, 2000; Nusser, 2009). Action potentials (APs) traveling from the axon hillock to the presynaptic terminals cause the brief opening of voltage-gated calcium channels (VGCCs) and a subsequent rush of $\mathrm{Ca}^{2+}$ in presynaptic terminals. At most presynaptic terminals, $\mathrm{Ca}^{2+}$ influx was shown to be mainly mediated by high-voltage activated $\mathrm{Ca}^{2+}$ channels, namely P/Q-, N-, and R-type VGCCs (Luebke et al., 1993; Takahashi and Momiyama, 1993; Wu and Saggau, 1995; Wu et al., 1999; Ishikawa et al., 2005; Li et al., 2007). Although P/Q- and $\mathrm{N}$-type VGCCs mediate fast synchronous neurotransmitter release, their specific contribution to $\mathrm{Ca}^{2+}$ signaling and glutamate release remains to be elucidated.

The spatiotemporal dynamics of $\mathrm{Ca}^{2+}$ elevations associated with specific types of VGCCs remain largely unknown. Modeling experiments and theoretical studies showed that the initial $\mathrm{Ca}^{2+}$ influx in a neuron causes a sharp increase in the nanodomain $\mathrm{Ca}^{2+}$ concentration, followed by a rapid equilibration in a micron-range domain of a lower concentration (Simon and Lli- 
nas, 1985). Therefore, the position of VGCCs relative to the $\mathrm{Ca}^{2+}$ sensor is a key element regulating neurotransmission. Indeed, it is suggested that the distribution of presynaptic VGCCs is synapsedependent and is thought to optimize the synapse function (Reid et al., 2003; Scimemi and Diamond, 2012). Therefore, the expression level of VGCCs does not necessarily dictate its efficacy on triggering neurotransmitter release (Ermolyuk et al., 2013). Rather, the position of the $\mathrm{Ca}^{2+}$ source relative to the $\mathrm{Ca}^{2+}$ sensor is a better predictor of release efficacy. For example, microdomain-coupling of a calcium source to a sensor has to be supported by several VGCCs, whereas a lower number of VGCCs suffices to trigger the release of nanodomain-coupled vesicles (Bertram et al., 1996; Eggermann et al., 2012).

Large mossy fiber (MF) terminals originating from granule cells synapsing on CA3 pyramidal cells (MF-CA3) control the flow of information from the dentate gyrus to the hippocampus proper. This central synapse possesses the features required for sparse coding of information. MFs form giant presynaptic terminals comprised of multiple release sites $(18-45)$ closely positioned on CA3 pyramidal cells apical dendrites (Amaral and Dent, 1981; Rollenhagen et al., 2007). This peculiar anatomical arrangement confers MFs with the ability to trigger APs in CA3 pyramidal cells in response to repetitive granule cell firing (Jung and McNaughton, 1993; Urban et al., 2001; Henze et al., 2002). The latter is made possible by the large short-term facilitation experienced by this synapse which ensures that granule cell firing results in CA3 pyramidal cell firing (Toth et al., 2000; Henze et al., 2002). Short-term facilitation at MF-CA3 synapses requires saturation of intraterminal $\mathrm{Ca}^{2+}$ buffer and operates by synchronization of multivesicular release and recruitment of additional release sites (Chamberland et al., 2014; Vyleta and Jonas, 2014).

At MF terminals, P/Q-, N-, and R-type VGCCs contribute a different fraction of the total $\mathrm{Ca}^{2+}$ influx in the presynaptic terminal (Li et al., 2007) and possess different functions (Dietrich et al., 2003). Whereas R-type VGCCs control long-term potentiation at these synapses, fast synchronous release of glutamate is under the control of P/Q- and N-type VGCCs (Castillo et al., 1994; Dietrich et al., 2003; Pelkey et al., 2006; Li et al., 2007). Intriguingly, although P/Q- and N-type VGCCs possess similar biophysical properties ( $\mathrm{Li}$ et al., 2007), MF terminals use both types. Currently it is not known whether P/Q- and N-type VGCCs fulfill specialized functions during short-term facilitation.

Overall, our findings demonstrate the functional specialization of P/Q- and N-type VGCCs in controlling synchronous glutamate release and short-term facilitation. N-type VGCCs ensure transmission at few release sites through spatially heterogeneous $\mathrm{Ca}^{2+}$ elevations and favor synchronization of multivesicular release. In contrast, P/Q-type VGCCs operate at more release sites through microdomain signaling and enhance short-term facilitation by recruiting additional release sites through spatially homogeneous $\mathrm{Ca}^{2+}$ elevations.

\section{Materials and Methods}

Hippocampal slice preparation and electrophysiology. All experiments involving animals were performed in accordance with the Université Laval guidelines for animal welfare. Transverse hippocampal slices were prepared from wild-type C57BL/6 mice (P17-P25) of either sex as described previously (Chamberland et al., 2014). Briefly, the animal was anesthetized and decapitated. The intact brain was rapidly collected and placed in oxygenated ice-cold ACSF containing the following (in mM): $87 \mathrm{NaCl}$, $25 \mathrm{NaHCO}_{3}, 2.5 \mathrm{KCl}, 1.25 \mathrm{NaH}_{2} \mathrm{PO}_{4}, 7 \mathrm{MgCl}_{2}, 0.5 \mathrm{CaCl}_{2}, 25$ glucose, and 75 sucrose, pH 7.4, $330 \mathrm{mOsm}$. Slices were cut on a Leica VT1000S Vibratome and transferred to a heated $\left(32^{\circ} \mathrm{C}\right)$ and oxygenated solution that contained the following (in mM): $124 \mathrm{NaCl}, 25 \mathrm{NaHCO}_{3}, 2.5 \mathrm{KCl}, 1.2$
$\mathrm{MgCl}_{2}, 2.5 \mathrm{CaCl}_{2}$, and 10 glucose, $\mathrm{pH} 7.4,300 \mathrm{mOsm}$. Slices were allowed to recover from the slicing procedure for $30 \mathrm{~min}$, at which point the incubator was turned off and slices were left at room temperature for the duration of the experiments. Recordings were started $1 \mathrm{~h}$ following the recovery period.

Electrophysiology. Slices were carefully positioned in a recording chamber under an upright microscope equipped with a $40 \times$ water-immersion objective (Olympus). During experiments, slices were perfused $(2 \mathrm{ml} /$ min) with ACSF saturated with a gas mixture of $95 \% \mathrm{O}_{2}$ and $5 \% \mathrm{CO}_{2}$. The ACSF contained the following (in mM): $124 \mathrm{NaCl}, 25 \mathrm{NaHCO}_{3}, 2.5$ $\mathrm{KCl}, 1.2 \mathrm{MgCl}_{2}, 2.5 \mathrm{CaCl}_{2}, 10$ glucose, 0.001 bicuculine methiodine, $\mathrm{pH}$ 7.4, $300 \mathrm{mOsm}$. For experiments performed in physiological calcium condition, the $\mathrm{CaCl}_{2}$ concentration was lowered to $1.2 \mathrm{~mm}$ and $\mathrm{MgCl}_{2}$ was adjusted to $2.5 \mathrm{~mm}$. All electrophysiological and calcium imaging experiments were performed at $32-34^{\circ} \mathrm{C}$. Visually guided whole-cell recordings were obtained from CA3 pyramidal cells with 3-4 M $\Omega$ borosilicate pipettes. The intracellular solution contained the following (in mM): 120 K-gluconate, $20 \mathrm{KCl}, 10$ HEPES, $2 \mathrm{MgCl}_{2}, 2 \mathrm{Mg}_{2} \mathrm{ATP}, 0.3$ NaGTP, 7 phosphocreatine, 0.6 EGTA, pH 7.2, 295 mOsm. In voltageclamp experiments, the neurons were held at $-70 \mathrm{mV}$. The electrophysiological signal was amplified and low-pass filtered at $2 \mathrm{kHz}$ with an Axopatch 200B or Multiclamp 700B (Molecular Devices), digitized at 10 $\mathrm{kHz}$ with a Digidata $1322 \mathrm{~A}$ or Digidata $1440 \mathrm{~A}$ (Molecular Devices) and recorded on a personal computer using the Clampex 9.0 and 11.0 software (Molecular Devices). MF EPSCs were evoked by brief $(0.1 \mathrm{~ms})$ electrical stimulation of the MF tract in stratum lucidum using a glass pipette connected to an electrical stimulator (A360, WPI). Recording epochs consisted of bursts of 10 stimuli evoked at $20 \mathrm{~Hz}$. These epochs were repeated every $30 \mathrm{~s}$ to avoid possible induction of long-term changes in synaptic strength. Series resistance was not compensated, but the access resistance and input resistance were monitored in every epoch with a 10 $\mathrm{mV}$ hyperpolarizing step. If the access or input resistance varied by $>15 \%$, cells were discarded from further analysis.

Random-access two-photon microscopy. Two-photon calcium imaging was performed using a custom built random-access multiphoton (RAMP) microscope controlled with a software written in LabVIEW (Otsu et al., 2008). The laser beam coming from a femtoseconds titanium:sapphire Coherent Chameleon Ultra II ( $80 \mathrm{MHz}, 140 \mathrm{fs}$, average power $>3.5 \mathrm{~W}$, Coherent) was tuned to $800 \mathrm{~nm}$ to excite simultaneously the morphological dye and the calcium indicator. The laser beam was directed through a pair of acousto-optic deflector (AODs, A-A OptoElectronics). These devices provide non-mechanical redirection of the laser beam in the XY direction, allowing ultrafast deflection of the laser and therefore multisite recording. The sample was illuminated by focusing the laser beam through a $25 \times$ water-immersion objective $(\mathrm{NA}=$ 0.95 , Leica). Transmitted fluorescence was collected with an oil condenser $(\mathrm{NA}=1.4)$ and low-pass filtered at $720 \mathrm{~nm}$. The emitted photons were separated in two channels (green calcium indicator and red morphological dye) using a $580 \mathrm{~nm}$ dichroic mirror (Semrock). Photons directed to the green channel were bandpass filtered at 500-560 nm and photons in the red channel were bandpass filtered at 595-665 nm (Semrock). Photons in the green and red channels were counted with two independent external AsGaP Hamamatsu (H7422P-40) photomultiplier tube. Fluorescence measurements were acquired with software written in LabVIEW and saved on a personal computer.

Presynaptic calcium imaging. Whole-cell recordings were obtained from visually identified granule cells with borosilicate pipettes of 5-6 $\mathrm{M} \Omega$. The pipette solution contained the following (in $\mathrm{mM}$ ): $120 \mathrm{~K}$ gluconate, $20 \mathrm{KCl}, 10 \mathrm{HEPES}, 2 \mathrm{MgCl}_{2}, 2 \mathrm{Mg}_{2} \mathrm{ATP}, 0.3 \mathrm{NaGTP}, 7$ phosphocreatine, and 0.04 AlexaFluor-594. This recording solution was supplemented with Fluo-5F ( $385 \mu \mathrm{M}$; Life Technologies). For experiments looking at single AP evoked calcium transients and the effect of VGCCs blockade, we used the medium-affinity calcium indicator Fluo-5F to avoid the potential confounding effects of saturation by highaffinity indicators. Fluo-5F also provided a sufficient signal-to-noise ratio to explore the effect of toxins with minimal number of scans.

Following break-in, the morphological indicator and the calciumsensitive dye were allowed to passively diffuse in the axon for $1 \mathrm{~h}$ before beginning recording. This was essential to ensure proper equilibration of 
the $\mathrm{Ca}^{2+}$ indicator (Scott and Rusakov, 2006). The axon of an individual granule cell was tracked to CA3 using AlexaFluor-594 fluorescence, where giant MF boutons could be easily identified based on their unique morphology (Fig. 1A, insets). Points (30-54) were positioned on the MF bouton, such as to obtain recordings from many calcium microdomains in the whole structure with as little overlap as possible (Fig. $1 B, E$; Chamberland et al., 2014). The dwell time for each point was $50 \mu$ s and the laser was repositioned between locations in $\sim 6.5 \mu \mathrm{s}$. This resulted in a recording frequency of $339-617 \mathrm{~Hz}$. Optical recordings were triggered by a transistor-transistor logic (TTL) signal sent from the digitizer to the optical acquisition system. The boutons were illuminated as little as possible, but sufficiently to obtain the full course of the calcium transients. Recording duration was therefore adjusted to $2 \mathrm{~s}$. Recordings were repeated every $15 \mathrm{~s}$. Under these conditions, a single AP evoked at the soma reliably evoked calcium transients in MF boutons, without failures (Fig. $1 C, D, F, G$; Scott and Rusakov, 2006; Chamberland et al., 2014).

To observe the effect of blocking P/Q- or N-type VGCCs on presynaptic calcium transient amplitude, recording epochs consisted of 20 sweeps of $1 \mathrm{AP}$, evoked at $0.1 \mathrm{~Hz}$. Recordings were continued during toxin application for $10 \mathrm{~min}$, after which 20 sweeps were acquired to analyze the toxin's effect. Long-term recordings of calcium elevations in boutons were performed with automatic repositioning of the points following each epoch. Points were tagged with a physical coordinate based on the structure of the recorded bouton. The repositioning was performed based on a $z$-stack of the AlexaFluor-594 fluorescence. During acquisition, recordings were systematically checked for stability by comparing the fluorescence intensity of the morphological dye between trials.

Experiments were terminated if the following signs of photodamage were observed: the decay time constant of the calcium transient became longer, the structure became permanently green and would not revert to baseline fluorescence, the green baseline fluorescence fluctuated $>20 \%$, or APs no longer evoked detectable $\mathrm{Ca}^{2+}$ transients. If these conditions appeared, no boutons from the same axon were recorded and the neuron was discarded. Recordings were discarded if the baseline morphological dye fluorescence varied by $>20 \%$ for single points following toxins application.

Pharmacology. Highly selective toxins were used to dissect the roles of VGCCs. Ca 2.1 (P/Q-type) VGCCs were blocked with $200 \mathrm{~nm} \omega$-Agatoxin IVA (AgTx, Alomone Labs). $\mathrm{Ca}_{\mathrm{V}} 2.2$ (N-type) VGCCs were antagonized with $200 \mathrm{~nm} \omega$-conotoxin GVIA (CTx; Alomone Labs). A submicromolar concentration of CTx was used to enforce CTx selectivity for N-type VGCCs (Tringham et al., 2008). For all electrophysiological and calcium imaging experiments involving AgTx and CTx, toxins were perfused for a period of $10 \mathrm{~min}$ to ensure proper diffusion of toxins and full blockade of VGCCs. Toxins were diluted in water, aliquoted, and kept in the freezer until used. The slow calcium chelator EGTA-AM (100 $\mu \mathrm{M}$, Anaspec) was aliquoted in DMSO (final concentration of DMSO in ACSF $=0.1 \%$ ) and kept in the freezer. The MF origin of EPSCs was assessed by their fast rise phase, large amplitude, and extensive short-term facilitation during repetitive stimulation. (2S, $\left.2^{\prime} \mathrm{R}, 3^{\prime} \mathrm{R}\right)-2-\left(2^{\prime}, 3^{\prime}\right.$-Dicarboxycyclopropyl)glycine (DCGIV , $1 \mu \mathrm{M}$ ) was applied after experiments ( 41 neurons/71 neurons) to confirm MF identity of EPSCs. DCG-IV application diminished control EPSC amplitude by $89 \pm 1.9 \%(n=41)$.

Electrophysiological data analysis. Electrophysiological recordings were analyzed using Clampfit 10.2 (Molecular Devices). The amplitude of EPSCs was measured at the peak. EPSC amplitudes were normalized to the amplitude of the first EPSC. Values are expressed as mean \pm SEM throughout the paper and in the figures, except for quantal parameters obtained using variance-mean analysis (see Figs. 3 and 4), which show mean \pm SD. Paired or unpaired Student's $t$ tests were used to determine whether the differences observed were statistically significant. The significancelevel was set at $p<0.05$. The $p$ values are reported as follow throughout the paper and the figures: ${ }^{*} p<0.05,{ }^{* *} p<0.01,{ }^{* *} p<0.001$.

Coefficient of variation analysis. Coefficient of variation $(\mathrm{CV})$ analysis is a statistical method based on the binomial model of neurotransmitter release. This assumption holds at the MF-CA3 synapse (von Kitzing et al., 1994). Changes in synaptic variance reflect the mechanisms underlying modifications in synaptic strength (Bekkers and Stevens, 1990; Malinow and Tsien, 1990; Faber and Korn, 1991; Larkman et al., 1997).
Analysis of the CV as a function of EPSC amplitude allows us to determine whether variations in synaptic strength originate from changes in the quantal size or in the number of active release sites. Graphical interpretation of the CV analysis is based on whether a change in EPSC amplitude is associated with changes in the CV. For facilitation, an increase in EPSC amplitude without changes in CV will report data points close to the horizontal $y=1$ line, indicating a change in quantal size. An increase in EPSC amplitude associated with a reduction in the CV will report data points on the diagonal line $y=x$, indicating an increase in the number of active release sites. This qualitative approach yields information on the mechanism involved in changes in synaptic strength, but is limited on the quantitative side. Indeed, CV analysis does not account for intrasite or intersite variance. In addition, CV analysis could be compromised if release probability across release sites could vary freely and/or in opposite direction. In the particular case of the MF terminals, release probability are very likely to vary in the same direction, because of: (1) all release sites at the MF terminal are encompassed in a single giant structure (Rollenhagen et al., 2007), (2) the extensive short-term facilitation observed during long train of stimuli (Toth et al., 2000; Chamberland et al., 2014), (3) loose-coupling between $\mathrm{Ca}^{2+}$ sources and sensors (Vyleta and Jonas, 2014), and (4) cooperation between $\mathrm{Ca}^{2+}$ domains in the structure (Stanley, 2015). The CV of EPSCs was measured from all sweeps recorded, except those contaminated with spontaneous events which were discarded from the analysis.

Covariance analysis. To measure the quantal size associated with successive EPSCs in the trains, covariance analysis was used (Scheuss et al., 2002). This method assumes that intervals between trains are sufficiently long so the synapse is in the same state for each train of stimuli and a binomial model of synaptic transmission (von Kitzing et al., 1994). Furthermore, under our experimental conditions, there is no postsynaptic contribution to short-term facilitation (Chamberland et al., 2014). We assume that there is no postsynaptic contribution to the covariance. This statistical analysis possess the advantages of monitoring synaptic parameters for individual responses during trains of stimuli, and not just the global parameters measured for the full trains. It also accounts for quantal variability and heterogeneity in the release probability between release sites. Quantal variability is likely to occur at the MF synapse, considering the large variance between the sizes of active zones measured at the ultrastructural level (Rollenhagen et al., 2007), and the possibility of multivesicular release at lower release probability (Chamberland et al., 2014). Furthermore, the release sites possess nonuniform release probability, with some release sites recruited later in repetitive trains of activity. This statistical approach also possesses limitations. Determination of the number of release sites with covariance analysis may not be so reliable in presence of intersite quantal variance, and is only robust when the probability of release is very high. We therefore used this method to measure the quantal size associated with individual EPSCs during trains.

The covariance between successive EPSCs was calculated using this formula (Scheuss et al., 2002):

$$
\operatorname{Cov}_{i, i+1}=\frac{1}{\mathrm{R}-1} \sum_{r=1}^{R-1}\left(I_{i, r}-I_{i, r+1}\right)\left(I_{i+1, r}-I_{i+1, r+1}\right) / 2,
$$

with $I$ representing single EPSC amplitude, $i$ the position of the EPSC in train, $R$ the total number of train recorded, and $r$ the train number. The variance between EPSCs at the same position in successive trials was calculated as follows:

$$
\operatorname{Var}_{i}=\frac{1}{\mathrm{R}-1} \sum_{r=1}^{R-1}\left(I_{i, r}-I_{i, r+1}\right)^{2} / 2 .
$$

From this, the quantal size $(Q)$ was estimated as follows:

$$
Q_{i}=\frac{\operatorname{Var}_{i}}{\overline{\bar{I}}_{i}}-\frac{\operatorname{Cov}_{i, i+1}}{\bar{I}_{i+1}},
$$

with $\bar{I}$ representing the average EPSC amplitude.

Nonstationary variance-mean analysis. The quantal size and the number of active release sites were measured using nonstationary variancemean analysis (Meyer et al., 2001) and accounted for intrasite or intersite 

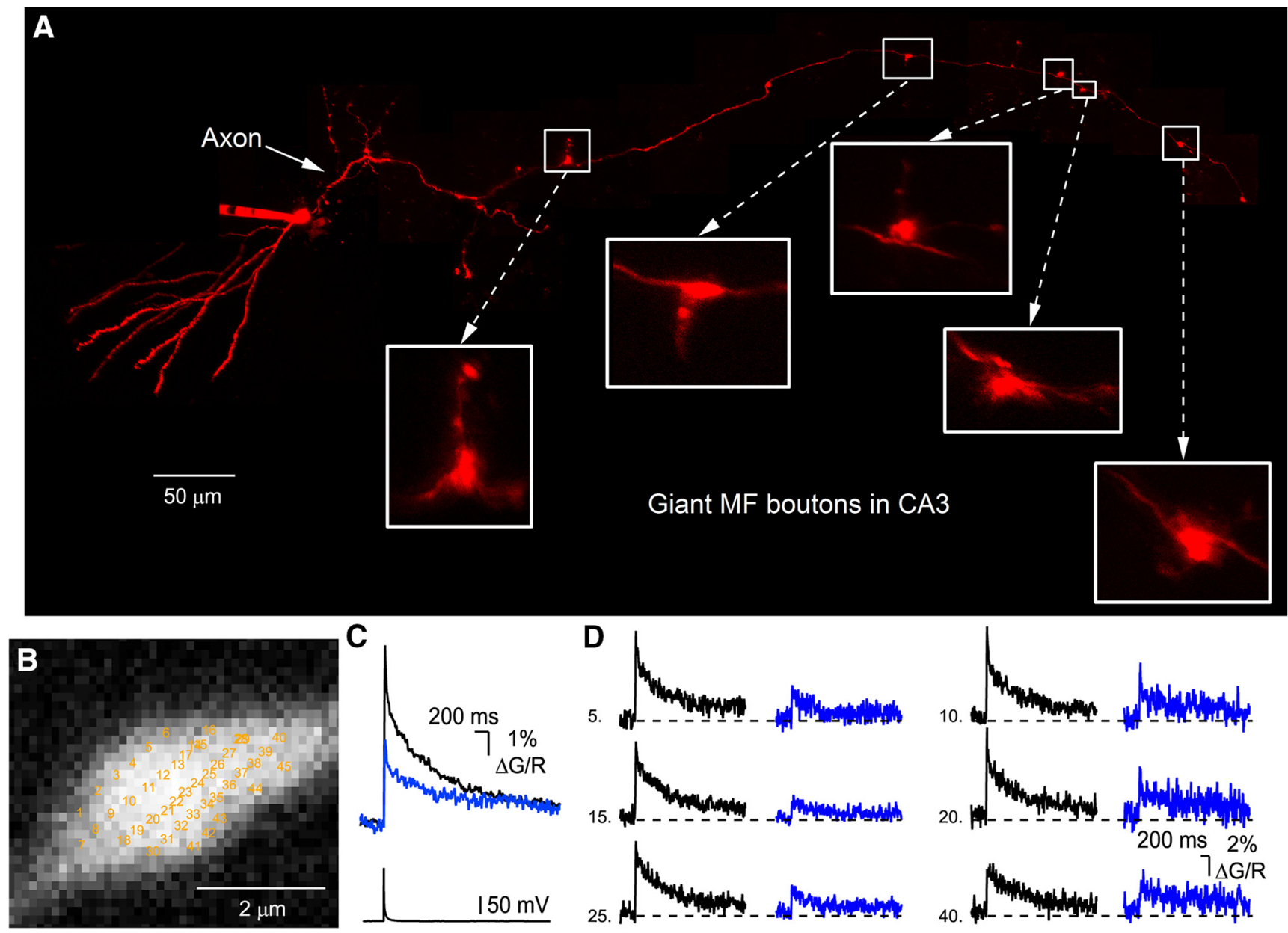

D.

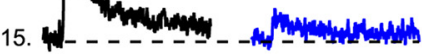
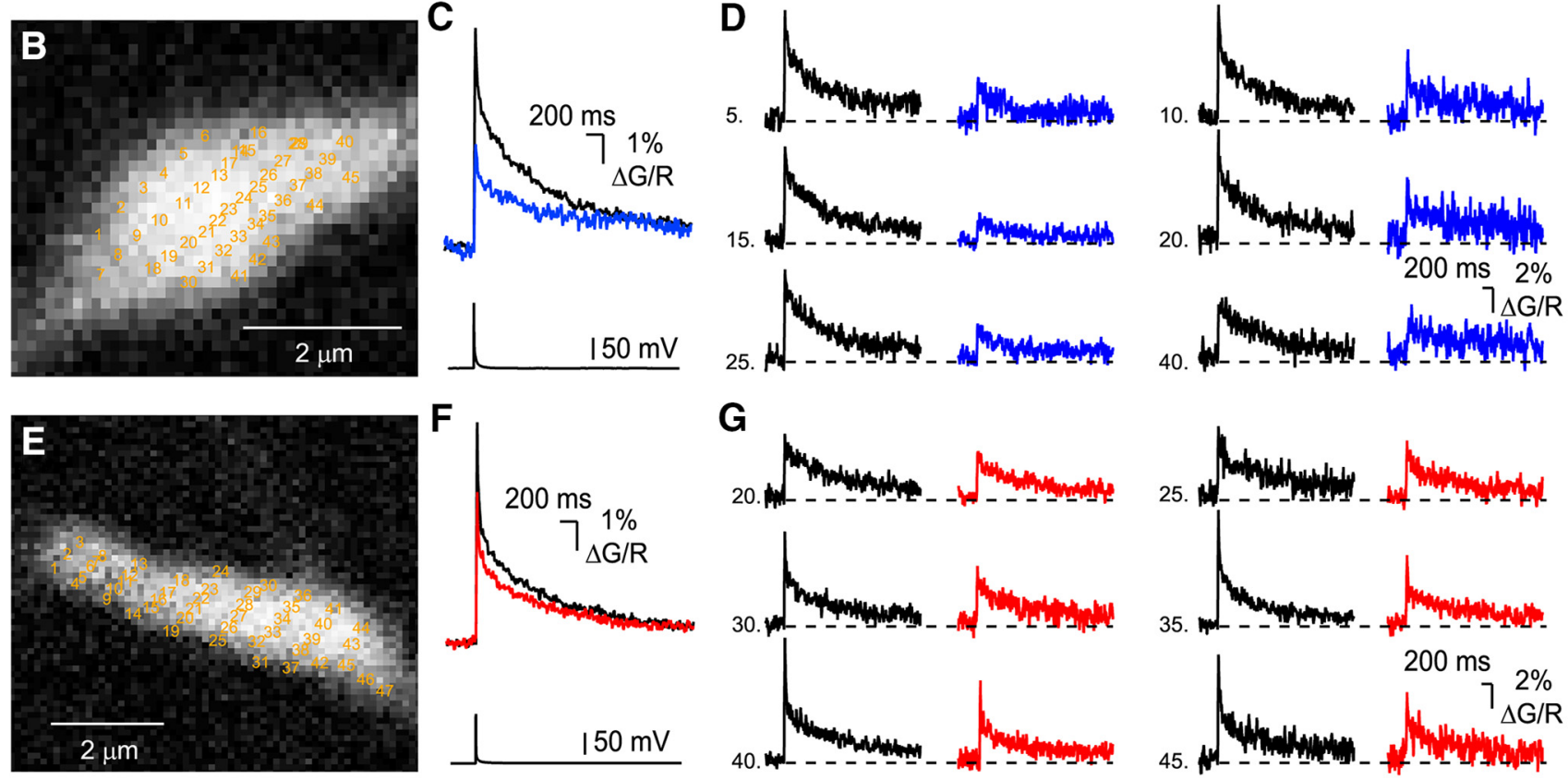

G
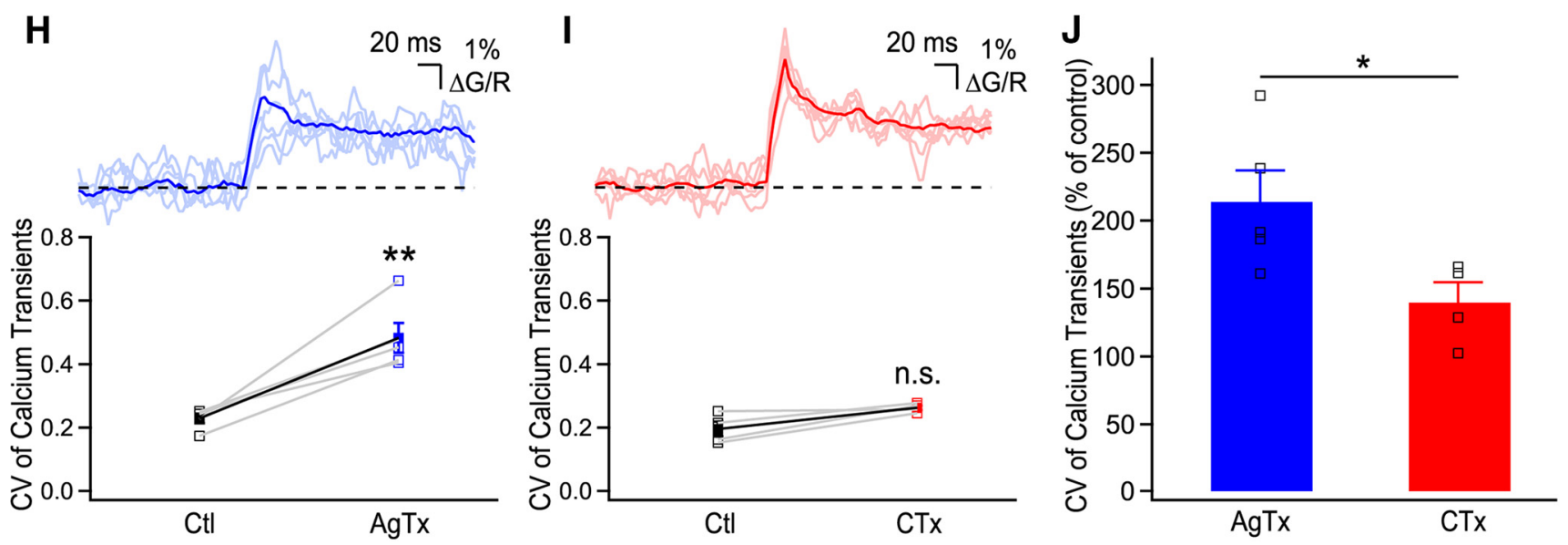

Figure 1. Distinct spatial profiles of $\mathrm{Ca}^{2+}$ elevations generated by P/Q- or N-type VGCCs. A, Photomontage of two-photon z-stack maximal projections showing an intact AlexaFluor-594-filled granule cell axonal arbor projecting to the CA3 region. $\boldsymbol{B}$, Expanded large MF boutons showing the recording sites (yellow numbers) for RAMP Ca ${ }^{2+}$ imaging. (Figure legend continues.) 
variances, and nonuniform release probabilities across release sites (Frerking and Wilson, 1996; Clements and Silver, 2000). We assumed a mixed contribution of intrasite and intersite variance for the following anatomical and functional reasons. First, the hypothesis of intersite variance is likely to hold, because the quantal size is likely to vary between release sites, as the size of the release sites is nonuniform in MF terminals (Rollenhagen et al., 2007) and may support the release of different number of vesicles. Second, intrasite variance is a likely contributor to the total variance, as the parabolic relationship between variance and mean does not terminate on the $x$-axis of the variance-mean plots (Frerking and Wilson, 1996; Clements and Silver, 2000; Silver, 2003). Furthermore, intrasite variance is a strong possibility given the presence of multivesicular release under lower release probabilities (Chamberland et al., 2014). To estimate the average quantal parameters $\mathrm{Q}$ and $\mathrm{N}$ during trains, the variance-mean plots were fitted with the following formula:

$$
y=\left[Q \bar{x}-\frac{Q \bar{x}^{2}(1+\alpha)}{\bar{x}+N Q \alpha}\right]\left(1+C V_{\text {inter }}^{2}\right)+Q \bar{x} C V_{\text {intra }}^{2},
$$

with $y$ representing the variance, $\bar{x}$ the average EPSC amplitude and $\alpha$ a parameter related to the nonuniformity of the release probability across release sites. We considered highly nonuniform release probabilities across release sites ( $\alpha=10$; Clements and Silver, 2000; Silver, 2003), as during trains, additional release sites are recruited to support short-term facilitation at the MF synapse (Chamberland et al., 2014). The total CV was experimentally measured as 0.45 . A $50 \%$ mixed contribution of intersite and intrasite variance was assumed for reasons explained above with $\mathrm{CV}_{\text {intra }}$ and $\mathrm{CV}_{\text {inter }}$ calculated as follows:

$$
C V_{\text {total }}^{2}=C V_{\text {intra }}^{2}+C V_{\text {inter }}^{2} \text {. }
$$

We estimated the maximum error on the quantal parameters associated with incorrect assumption of the variance location. For a given parameter, this was obtained by calculating the largest difference between the mixed intrasite and intersite variance and exclusive intrasite or intersite. This difference was expressed as a percentage of the values obtained using the mixed intrasite and intersite variance.

Calcium imaging data analysis. Optical data were extracted using a routine written in LabVIEW. The data were transferred to Igor Pro 6.3 (Wavemetrics). The absolute peak calcium transient amplitude was measured from the average of 20 trials. The peak amplitude of calcium transients was measured in Igor Pro. Due to the temporal resolution $(339-617 \mathrm{~Hz})$ of the imaging paradigm, we estimate the maximal error associated with measurement of the peak amplitude to be $9 \%$ at the slowest sampling rate. The spatial heterogeneity of calcium elevations recorded in multiple sites was assessed by measuring the increase in SE of all points at the level of the peak amplitude. The symbol (yellow numbers) identifying the recording locations are intentionally large for better visibility (Fig. $1 B, E$ ). The underlying volume physically excited by the laser is not indicated quantitatively. To compare changes in spatial heterogeneity in control condition and following toxin treatment independently of the change in peak amplitude, the $\mathrm{CV}$ was measured. The axial point-spread function (PSF) of the imaging system was measured using subresolution fluorescent beads and found to be $600 \mathrm{~nm}$. This spatial

$\leftarrow$

(Figure legend continued.) Note that the yellow numbers are intentionally large to be readable. C, Bouton-averaged $\mathrm{Ca}^{2+}$ elevations recorded in $\boldsymbol{B}$ evoked by a single $\mathrm{AP}$ in control (black) and in the presence of $\mathrm{AgTx}$ (blue). D, Examples of single voxels $\mathrm{Ca}^{2+}$ elevations in control condition and following application of AgTx. Traces shown are the average of 20 trials. E, Recording sites are indicated on a large $M \boldsymbol{M F}$ terminal for data shown in $\boldsymbol{F}$ and $\boldsymbol{G}$. $\boldsymbol{F}$, Bouton-averaged calcium elevations recorded in control (black) and in presence of $(\mathrm{Tx}$ (red), and corresponding singlevoxel examples (G) in control (black) and in (Tx (red). Traces are average of 20 trials. $\boldsymbol{H}$, Top, Traces of single voxel examples shown in $\boldsymbol{D}$, with their average overlaid. Note the large deviations from the average. Bottom, Plot showing the spatial $\mathrm{CV}$ of peak $\mathrm{Ca}^{2+}$ transients in control and following the application of AgTx. I, Top, Overlay of the traces shown in $\mathbf{G}$, with their average. Bottom, Plot showing the effect of $\mathrm{CTx}$ on the spatial $\mathrm{CV}$ of peak $\mathrm{Ca}^{2+}$ elevation (n.s. = nonsignificant; $p>0.05$ ). J, AgTx increases the spatial heterogeneity of $\mathrm{Ca}^{2+}$ elevations significantly more than CTx. Data represent mean \pm SEM. ${ }^{*} p<0.05,{ }^{* *} p<0.01$. resolution could potentially introduce error due to oversampling, if several recorded points have overlapping PSFs. To limit spatial oversampling, we used a two steps approach. First, care was taken to select points with little overlap in the bouton during recordings. Additional care was taken to cover the whole bouton with points to sample to whole structure. However, a few points $(9.7 \pm 2.6 \%, n=9$ boutons) showed significant overlap. These points were excluded from the analysis. It is noteworthy that points located very close to each other showed a quasiidentical signal, thereby confirming that our imaging approach is precise (data not shown). Second, only points that were at least $300 \mathrm{~nm}$ apart were analyzed. Under this condition, the extent to which one given point can contain overlapping signal with an adjacent point is limited by the overlapping PSFs. Considering a circular PSF of $300 \mathrm{~nm}$ radius, the area of two intersecting circles and the declining excitation on the edges of the PSF, two points separated by $300 \mathrm{~nm}$ should contain overlapping fluorescence of $<20 \%$. This value represents the maximal oversampling for two adjacent points in our recordings. This maximal oversampling condition was present in only $24.8 \pm 2 \%$ ( $n=9$ boutons) of points, therefore limiting the error introduced by a possible spatial oversampling of the bouton.

\section{Results}

\section{Specialized $\mathrm{Ca}^{2+}$ spatial dynamics through $\mathrm{P} / \mathrm{Q}$ - and N-type VGCCs}

$\mathrm{Ca}^{2+}$ elevations in MF boutons are spatially heterogeneous (Chamberland et al., 2014). How these heterogeneous $\mathrm{Ca}^{2+}$ microdomains are generated remains unknown. In large MF terminals, P/Q- and N-type VGCCs gate $\mathrm{Ca}^{2+}$ influx and mediate synchronous glutamate release (Castillo et al., 1994; Pelkey et al., 2006; Li et al., 2007). Therefore, specialized $\mathrm{Ca}^{2+}$ spatial dynamics through P/Q- and N-type VGCCs could support heterogeneous $\mathrm{Ca}^{2+}$ microdomains.

To record $\mathrm{MF} \mathrm{Ca}^{2+}$ elevations with the highest spatial and temporal resolution, we performed random-access two-photon $\mathrm{Ca}^{2+}$ imaging from unambiguously identified giant MF boutons (Fig. 1A). $\mathrm{Ca}^{2+}$ elevations were generated by single AP evoked at the level of the cell body by brief current injection. As previously reported under similar conditions of added exogenous buffer (Fluo-5F), $\mathrm{Ca}^{2+}$ elevations had a slow decay time (Fig. $1 C, F$ ), in part due to a low extrusion rate (Scott and Rusakov, 2006). We measured $\mathrm{Ca}^{2+}$ elevations from multiple diffraction-limited regions in MF terminals in control conditions and following the application of toxins (Fig. $1 B, E$ ). In control conditions, $\mathrm{Ca}^{2+}$ elevations recorded in MF boutons had highly heterogeneous amplitudes (Fig. 1D,G; Chamberland et al., 2014). Following $\omega$-agatoxin IVA (AgTx) application to block P/Q-type VGCCs (Fig. 1C), there was a significant increase in the CV of the peak $\mathrm{Ca}^{2+}$ transients recorded in different positions, indicating that $\mathrm{Ca}^{2+}$ elevations became more spatially heterogeneous after P/Qtype VGCCs blockade (Ctl: 0.229 \pm 0.02 , AgTx: $0.482 \pm 0.05 ; n=$ $5 ; p<0.01$; Fig. $1 H)$. On the other hand, application of CTx to block N-type VGCCs (Fig. $1 F$ ) failed to significantly increase the $\mathrm{CV}$ of $\mathrm{Ca}^{2+}$ elevations recorded in distinct subcompartments (Ctl: $0.195 \pm 0.02$, CTx: $0.262 \pm 0.01 ; n=4 ; p>0.05$; Fig. $1 I$ ). The increase in $\mathrm{CV}$ of $\mathrm{Ca}^{2+}$ transients following AgTx application was significantly larger than after perfusion of CTx (AgTx: $213.9 \pm 23.2 \%, n=5$; CTx: $139.7 \pm 14.9 \%, n=4 ; p<0.05$; Fig. $1 J)$. Therefore, these results indicate that following invasion of a single AP in the presynaptic terminal, $\mathrm{Ca}^{2+}$ influx through P/Qtype VGCCs is spatially more homogenous than $\mathrm{Ca}^{2+}$ influx through N-type VGCCs, and that N-type VGCCs contribute to the generation of heterogeneous $\mathrm{Ca}^{2+}$ microdomains. 


\section{Distinct roles of $\mathrm{P} / \mathrm{Q}$ - and N-type VGCCs in short-term facilitation}

Although P/Q- and N-type VGCCs share similar biophysical properties, these channels contribute differently to the spatial profile of $\mathrm{Ca}^{2+}$ elevations in MF terminals. These distinct $\mathrm{Ca}^{2+}$ dynamics could influence glutamate release. The powerful shortterm facilitation at MF-CA3 synapses involves the synchronization of multivesicular release and the recruitment of additional release sites (Chamberland et al., 2014). Therefore, we next chose to investigate whether P/Q- and N-type VGCCs possess distinct roles in short-term facilitation when granule cells fire repetitively.

Trains of 10 EPSCs evoked at $20 \mathrm{~Hz}$ (in the physiological firing frequency range for granule cells; Henze et al., 2002) were recorded in $2.5 \mathrm{~mm}$ external $\mathrm{Ca}^{2+}$. EPSCs were readily facilitated $\left(2.5 \mathrm{mM} \mathrm{Ca}^{2+}\right.$; first EPSC: $279 \pm 23.4 \mathrm{pA}$; 10th EPSC: $882.7 \pm$ $67.2 \mathrm{pA}, p<0.0001, n=36$; Fig. $2 A, B)$. Application of AgTx (first EPSC: $49.9 \pm 15.4 \mathrm{pA}$; 10th EPSC: $187.1 \pm 41.7 \mathrm{pA} ; n=16$; Fig. $2 A, B$ ) or CTx (first EPSC: $102.6 \pm 15.6$; 10th EPSC: $529.9 \pm$ 57.6; $n=19$; Fig. $2 A, B)$ strongly inhibited EPSC amplitude regardless of their position in the train, but neither toxin could block short-term facilitation. Coapplication of AgTx and CTx fully blocked release and short-term facilitation for trains of 10 stimuli (Fig. 2D,E). To address the specific roles of P/Q- and N-type VGCCs in short-term facilitation at MF-CA3 synapses, we used CV analysis (see Materials and Methods). In control condition, visual inspection of the $\mathrm{CV}$ analysis revealed a diagonal progression of the data points during trains, revealing that short-term facilitation results from the recruitment of additional release sites. Blocking N-type VGCCs resulted in a slight right shift of the data points, with a general trend close to diagonal (Fig. $2 C)$. Therefore, the recruitment of additional release sites was still active. In contrast, blockade of P/Q-type VGCCs fully prevented the recruitment of additional release sites, with the data points found close to a horizontal line (Fig. 2C). This shows that P/Qtype VGCCs are essential for the recruitment of additional release sites, whereas N-type VGCCs do not contribute to the recruitment of additional release sites. However, N-type VGCCs also contribute to short-term facilitation, likely through the synchronization of multivesicular release (Chamberland et al., 2014). Altogether, these data indicate that the specialized $\mathrm{Ca}^{2+}$ spatiotemporal dynamics gated by P/Q- and N-type VGCCs differently control short-term facilitation.

\section{$\mathrm{Ca}^{2+}$ influx through $\mathrm{P} / \mathrm{Q}$ - have access to more release sites than N-type VGCCs}

P/Q- but not N-type VGCCs promote the recruitment of additional release sites during short-term facilitation. This suggests that $\mathrm{Ca}^{2+}$ influx through P/Q-type VGCCs have access to more release sites than N-type VGCCs. Quantal parameters, including the number of active release sites, can be measured through statistical analysis of EPSC variance and mean (Clements and Silver, 2000; Scheuss et al., 2002).

To investigate whether P/Q-type VGCCs have access to more release sites and quantify the quantal parameters, we used two distinct approaches. First, we performed covariance analysis to measure the quantal size of each EPSC in the train, in control and in presence of toxins (Fig. $3 A-C$ ). This approach provides an estimate of $Q$ for each EPSC during a train in the presence of quantal variability (Scheuss et al., 2002). In control condition and in presence of CTx, the quantal size measured with covariance analysis was stable throughout the train $(n=35$ cells in control, $n=17$ cells in CTx; Fig. 3D). In contrast, the quantal size measured in presence of AgTx was gradually and significantly in- creased for the first five stimuli, confirming that short-term facilitation occurs through an increase in multivesicular release when N-type VGCCs alone mediate short-term facilitation $(n=$ 15 cells; Fig. 3D). To compare the quantal size between the three conditions after reaching steady-state, we pooled the last five points from the data plot. This analysis revealed that the quantal size in steady-state was significantly smaller in presence of CTx $(p<0.05$ vs Ctl and $p<0.05$ vs AgTx; Fig. $3 E)$. These data indicate that although P/Q-type VGCCs control more release sites, they are less efficient than N-type VGCCs at synchronizing multivesicular release.

Second, we used nonstationary variance-mean analysis to confirm the persistent decrease in Q throughout the train caused by CTx, and to estimate the number of active release sites (Meyer et al., 2001). We factored a mixed contribution of intersite and intrasite variability (see Materials and Methods) and accounted for differences in release probabilities between release sites (Clements and Silver, 2000; Silver, 2003). This statistical model allows determination of quantal parameters even in presence of variability between the release sites (Clements and Silver, 2000). Fitting the data points with a $\beta$ function revealed a parabolic relationship. Because the parabolic fit in variance-mean analysis is strongly distorted by changes in quantal size during trains, the quantal parameters were measured using only the steady-state portion of the quantal size for recordings performed in AgTx. Measuring quantal parameters under these assumptions revealed a larger number of active release sites in control $(N=19.6 \pm 5.6$; $n=12$; Fig. $3 F)$ and in CTx $(N=19.3 \pm 6.7 ; n=6$; Fig. $3 F)$ than in $\operatorname{AgTx}(N=4 \pm 2.5 ; n=9$; Fig. $3 F)$, indicating that P/Q-type VGCCs control a larger number of release sites. Because the number of release sites controlled by P/Q-type VGCCs closely resembles the total number of release sites, P/Q-type VGCCs could have access to all release sites by the end of the stimuli train. This possibility will be explored in the next section. As measured with covariance analysis, the quantal size was larger in control $(Q=$ $44.7 \pm 5.6 ; n=12$; Fig. $3 F)$ and in $\operatorname{AgTx}(Q=51.3 \pm 11.3 ; n=9$; Fig. $3 F)$ than in $\operatorname{CTx}(Q=33.7 \pm 3.7 ; n=6$; Fig. $3 F)$, highlighting the role of N-type VGCCs in controlling multivesicular release.

Although we assumed a mixed contribution of intersite and intrasite synaptic variance (Fig. 4C), these parameters cannot be directly measured in our system. To measure the error associated with incorrect assumption of the variance origin, we next simulated the effects of considering variance to be exclusively intrasite (Fig. 4A) or intersite (Fig. 4B). Consistent with previous studies, the intrasite or intersite variance did not affect the qualitative parabolic relationship of the variance to the mean (Frerking and Wilson, 1996). Furthermore, based on these simulations, we estimated that the error associated with incorrect assumption of the origin of variance was $<1 \%$ for the quantal size, and $<10.7 \%$ for the number of active release sites. Therefore, our results indicate that P/Q-type VGCCs have access to more release sites than $\mathrm{N}$-type VGCCs. On the other hand, N-type VGCCs are essential in controlling multivesicular release.

\section{$\mathrm{Ca}^{2+}$ influx through $\mathrm{P} / \mathrm{Q}$ - have access to release sites controlled by N-type VGCCs}

P/Q-type VGCCs generate spatially homogenous $\mathrm{Ca}^{2+}$ microdomains which are key in recruiting additional release sites during short-term facilitation. Are individual release sites under the exclusive control of one type of VGCCs, or are release sites controlled by a mixed contribution of $\mathrm{Ca}^{2+}$ influx through both P/Q- and N-type VGCCs? Neurotransmitter release can occur 
A

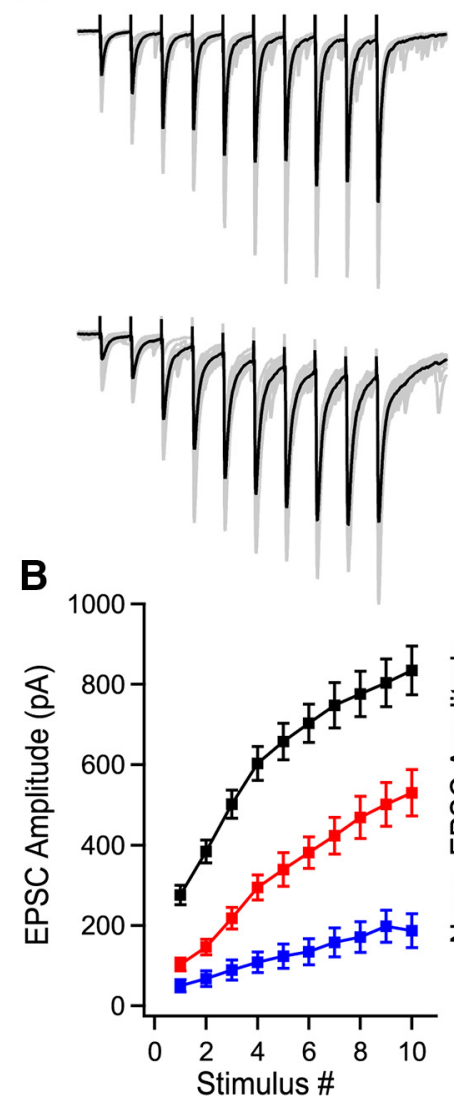

$+\mathrm{AgTx}$

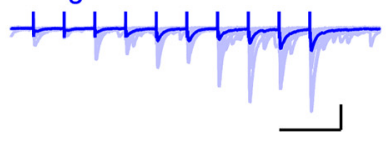

+ CTx

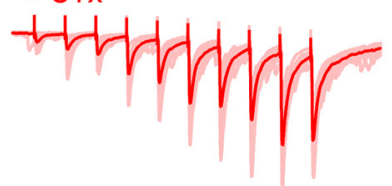

$150 \mathrm{pA}$

$100 \mathrm{~ms}$

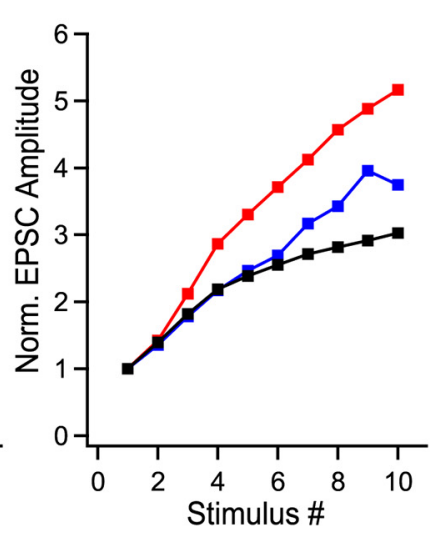

C

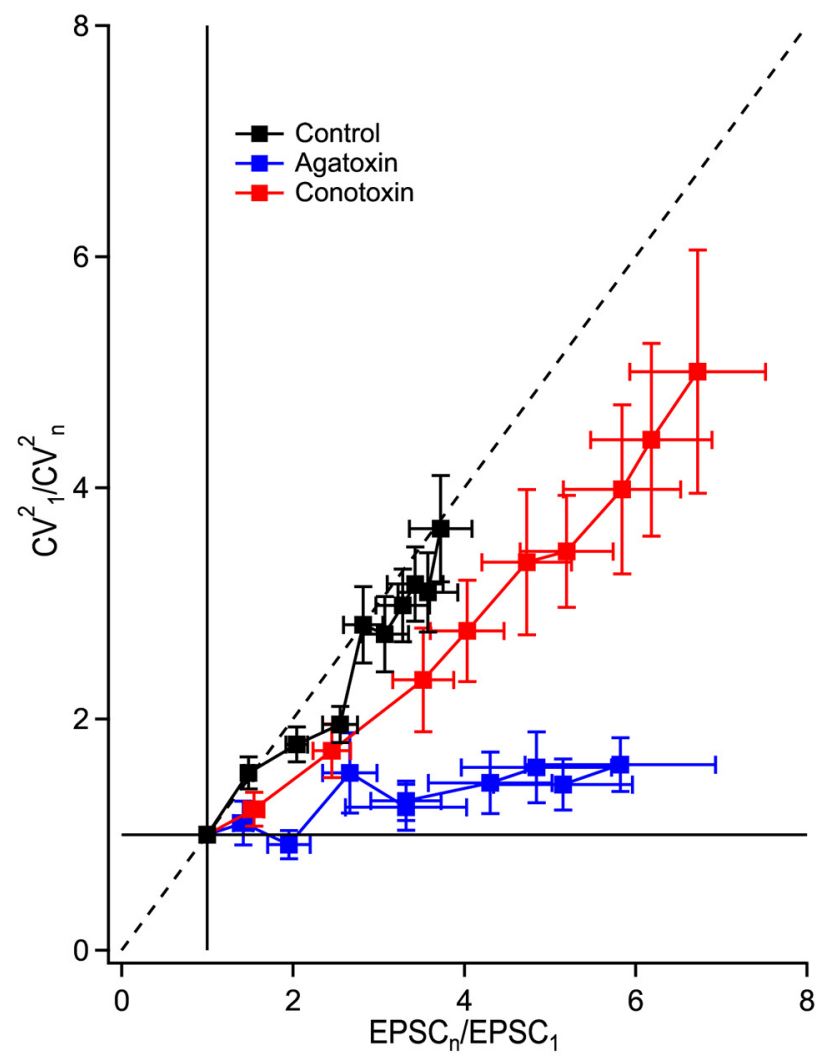

E

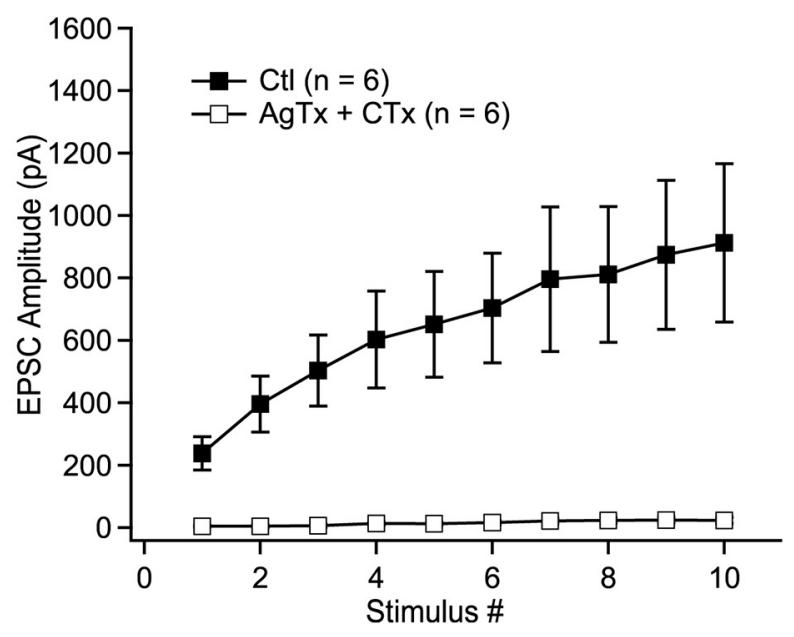

$100 \mathrm{~ms}$ $200 \mathrm{pA}$

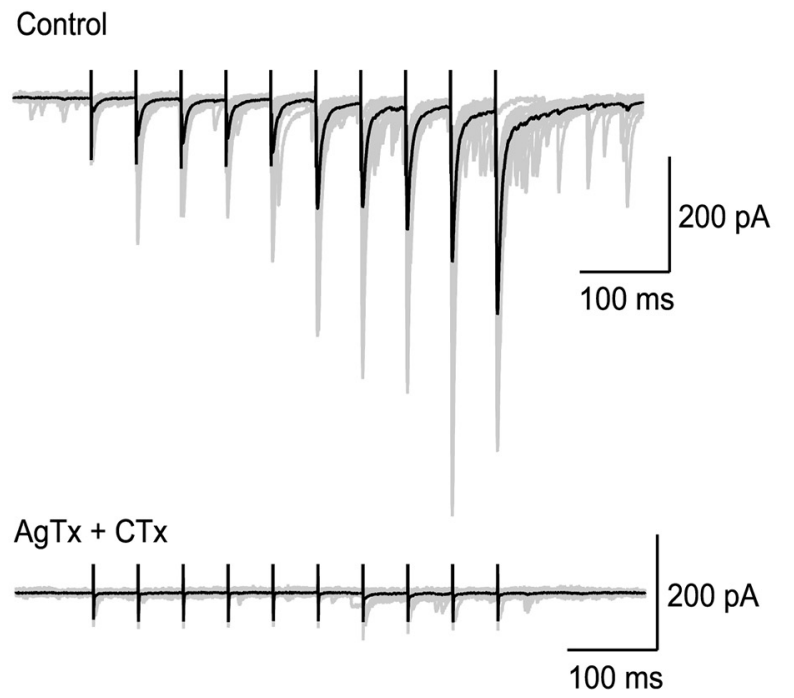

Figure 2. P/Q-type VGCCs contribute to short-term facilitation through recruitment of additional release sites and N-type VGCCs control multivesicular release. $A$, Trains of facilitating EPSCS evoked by 10 stimuliat $20 \mathrm{~Hz}(372.2 \pm 36.4 \%$ of first EPSC, $n=36, p<0.0001)$. Application of AgTx or CTx largely decreased EPSC amplitudes. $\boldsymbol{B}$, Summary plots of EPSC amplitude and normalized EPSC amplitude as a function of stimulus number. Normalized data were obtained by normalizing the averaged values. C, CV analysis identifies the mechanism supporting progression of EPSC amplitude during stimulation trains. Visual inspection of the CV graph reveals that P/Q- but not N-types VGCCs are essential to recruit additional release sites during trains of facilitating EPSCs. This is extracted from the position of the blue data points (AgTx) located close to the horizontal lines. On the other hand, EPSCs recorded in CTx are found close to the diagonal. Therefore, P/Q-type VGCCs are essential to recruit additional release sites during short-term facilitation, whereas $\mathrm{N}$-type VGCCs contribute to short-term facilitation through the synchronization of multivesicular release. The EPSC amplitude ( $x$-axis) was normalized for individual neurons before averaging. D, Example of EPSCs recorded in control conditions and following coapplication of AgTx and CTx. E, Summary plot showing the effect of combined application of AgTx and CTx on EPSC amplitude $(n=6)$. Data represent mean \pm SEM.

through $\mathrm{Ca}^{2+}$ entering the presynaptic terminal from a single VGCC (Weber et al., 2010; Scimemi and Diamond, 2012), through several VGCCs (Borst and Sakmann, 1996), and can result from combined influx through more than one type of
VGCC (Wheeler et al., 1994). When this last phenomenon occurs, blocking the different $\mathrm{Ca}^{2+}$ sources will show a supraadditive effect; the arithmetic sum of EPSC blockade will be superior to 100\% (Mintz et al., 1995; Reid et al., 2003). 
A

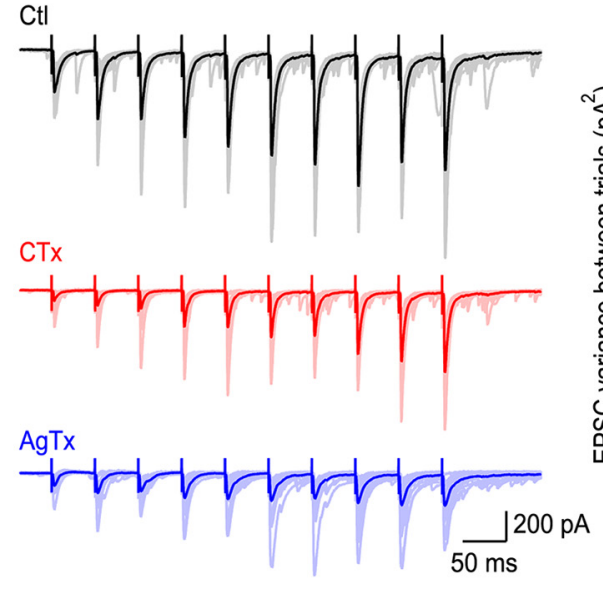

B

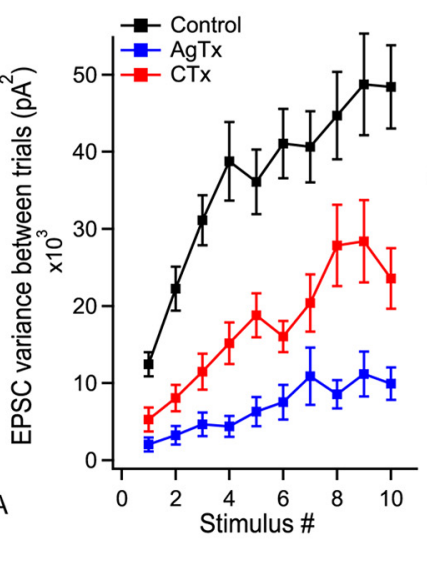

D

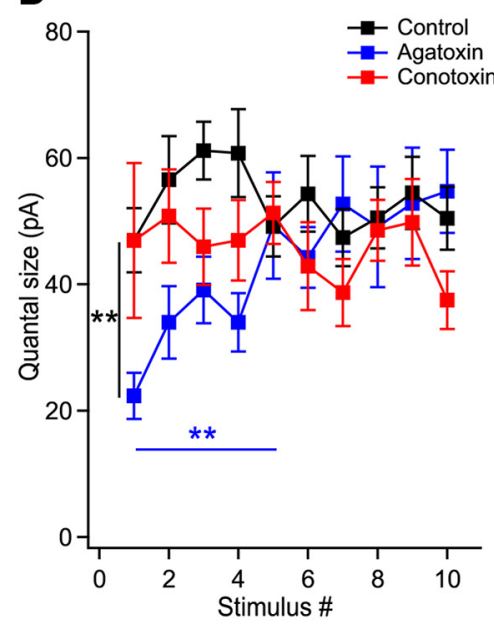

E

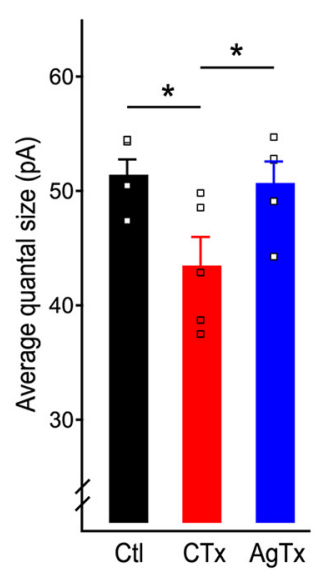

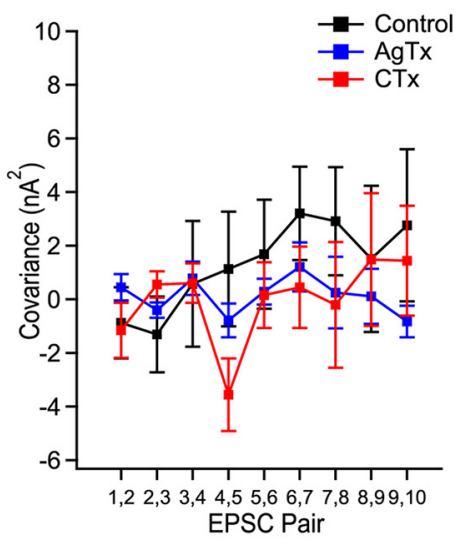

C
$\mathbf{F}$

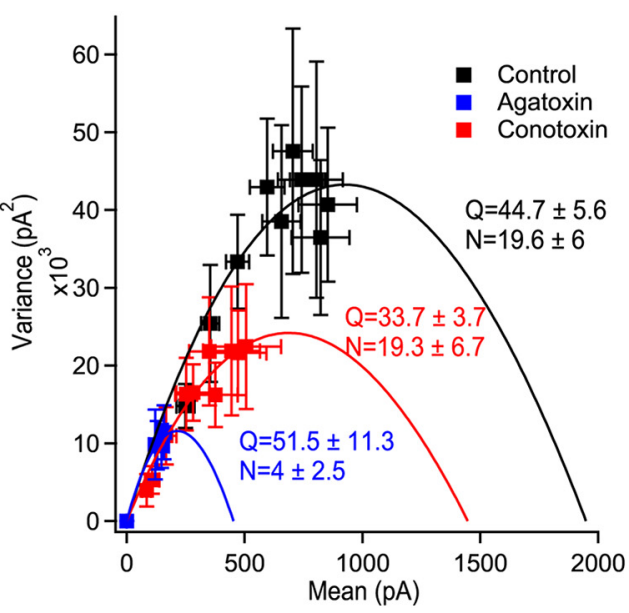

Figure 3. $\mathrm{Ca}^{2+}$ influx from P/Q-type VGCCs have access to more release sites than N-type VGCCs. A, Representative examples of EPSC trains recorded in control (black), in CTx (red), and in AgTx (blue). $\boldsymbol{B}$, Variance of pairs of EPSCs in consecutive trains for EPSCs recorded in control, CTx, and AgTx. C, Covariance between pairs of consecutive EPSCs in the train as a function of EPSC pair numbers. $\boldsymbol{D}$, Quantal size measured using covariance analysis as a function of stimulus number for EPSCs recorded in control, in the presence of AgTx, and in the presence of CTx. $\boldsymbol{E}$, Average quantal size in steady-state (last 5 stimuli) revealing a larger quantal size in control and in AgTx than in the presence of CTx. Squares represent data points of stimuli $6-10$ shown in $\boldsymbol{D}$. $\boldsymbol{F}$, Nonstationary variance-mean analysis accounting for a mixed contribution of intrasite and intersite variance identifies the quantal parameters for trains of EPSCs recorded in control and following blockade of P/Q- (blue) or N-type (red) VGCCs. Data represent mean \pm SEM. ${ }^{*} p<0.05,{ }^{* *} p<0.01$.

To address whether P/Q-type VGCCs have access to the same release sites as N-type VGCCs, we arithmetically summed the effects of AgTx and CTx on EPSC amplitude during trains of stimuli. The AgTx and CTx sum of effect was initially found above the $100 \%$ level, but demonstrated a marked and gradual decrease, reaching almost $100 \%$ at the end of the train (Fig. 5A). This indicates that under lower release probability, $\mathrm{Ca}^{2+}$ entering through P/Q- and N-type VGCCs have access to the same release sites. Interestingly, the sum of effect of AgTx and CTx did not reach the 100\% mark because of a similar reduction in the amount of short-term facilitation (Fig. $5 A-C$ ). Although the percentage of effect of AgTx was largely constant during the train, the percentage of effect of CTx on EPSC amplitude was gradually decreased (Fig. $5 \mathrm{C}$ ). This differential effect of toxins argues that the supra-additive effect is not due to the power law-dependency of neurotransmitter release on $\mathrm{Ca}^{2+}$ influx. More importantly, this result indicates that by the 10th stimulus, P/Q-type VGCCs may have access to the same vesicles as N-type VGCCs. In contract, N-type VGCCs do not appear to have access to all vesicles, even after a large number of stimuli.

To test this idea, we performed CV analysis to analyze the effect of the toxins on EPSCs throughout the train. We aimed to determine whether blocking P/Q- or N-type VGCCs had the same effect on the quantal parameters when release probability was increased through repetitive activity. For the first EPSC in the train, both toxins caused a reduction in the number of active release sites, as indicated by the data falling on the diagonal line (Fig. 5D). For subsequent stimuli in the train (5th and 10th shown), the effect of AgTx was purely a decrease in the number of active release sites. In contrast, CTx reduced the quantal size for the last stimulus in the train, because the data point moved closer to the horizontal line. Therefore, blocking N-type VGCCs initially reduced the number of active release sites, but eventually only decreased multivesicular release. This suggests that P/Qtype VGCCs have access to more release sites, including release sites controlled by N-type VGCCs. This idea is confirmed by two direct observations. First, the fraction of EPSCs blocked by CTx reached a maximum and plateaued by the fifth stimulus $(n=19$ cells; Figs. 5E1, red open markers, $2 B$ ). In contrast, the portion of EPSC blocked by AgTx gradually increased during the train $(n=$ 16 cells; Figs. 5E2, blue open markers, $2 B$ ). Altogether, these results indicate that N-type VGCCs trigger release at some active zones and actively control multivesicular release. In comparison, 
A

$$
\begin{aligned}
& \text { Intrasite Variance Only } \\
& \mathrm{CV}_{1}=0.45 \\
& \mathrm{CV}_{\| I}=0
\end{aligned}
$$
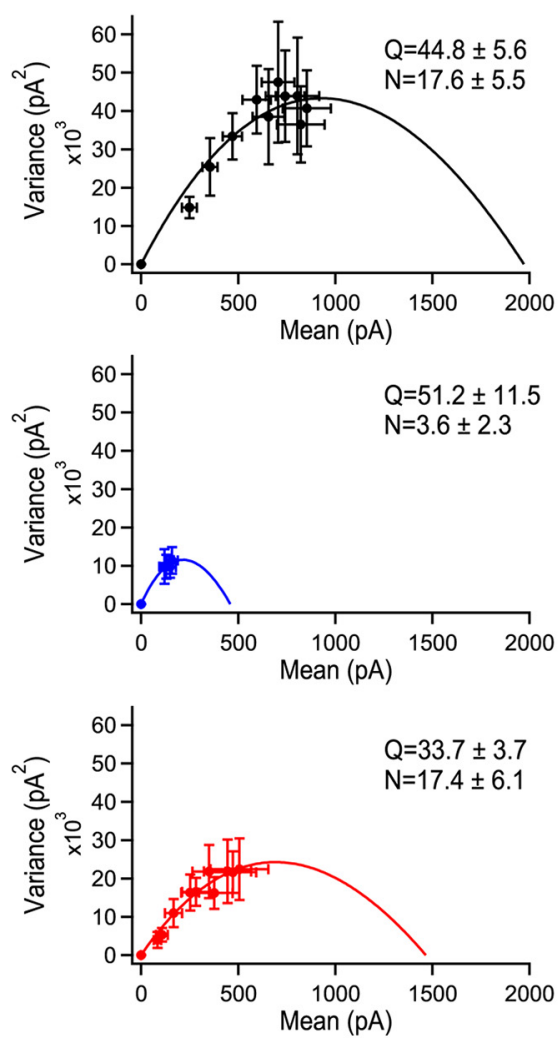

B

$$
\begin{aligned}
& \text { Intersite Variance Only } \\
& \mathrm{CV}_{1}=0 \\
& \mathrm{CV}_{\|}=0.45
\end{aligned}
$$
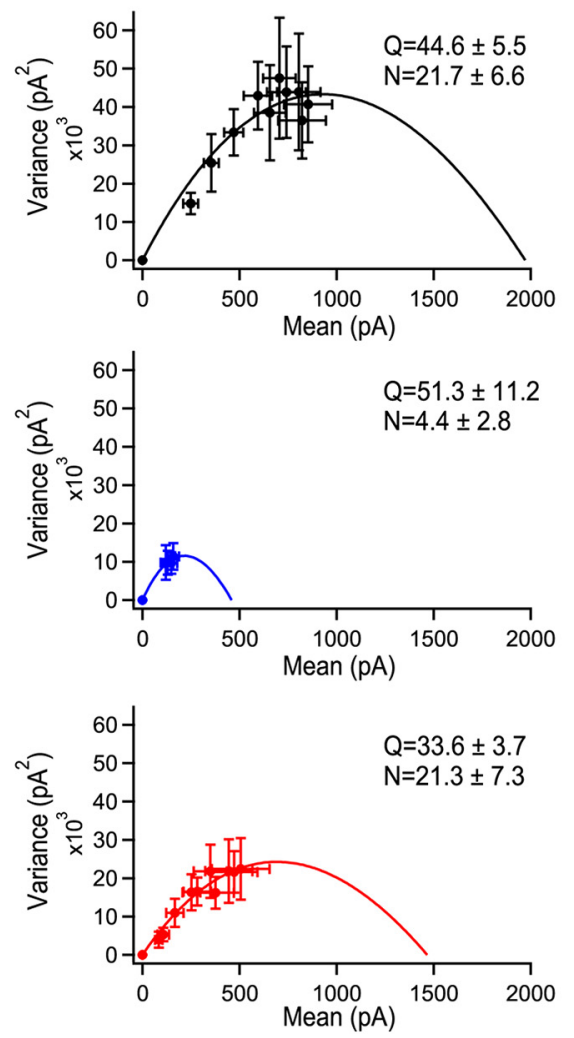

C

Mixed Intra- and Intersite Variance $\mathrm{CV}_{1}=0.318$

$\mathrm{CV}_{\|}=0.318$
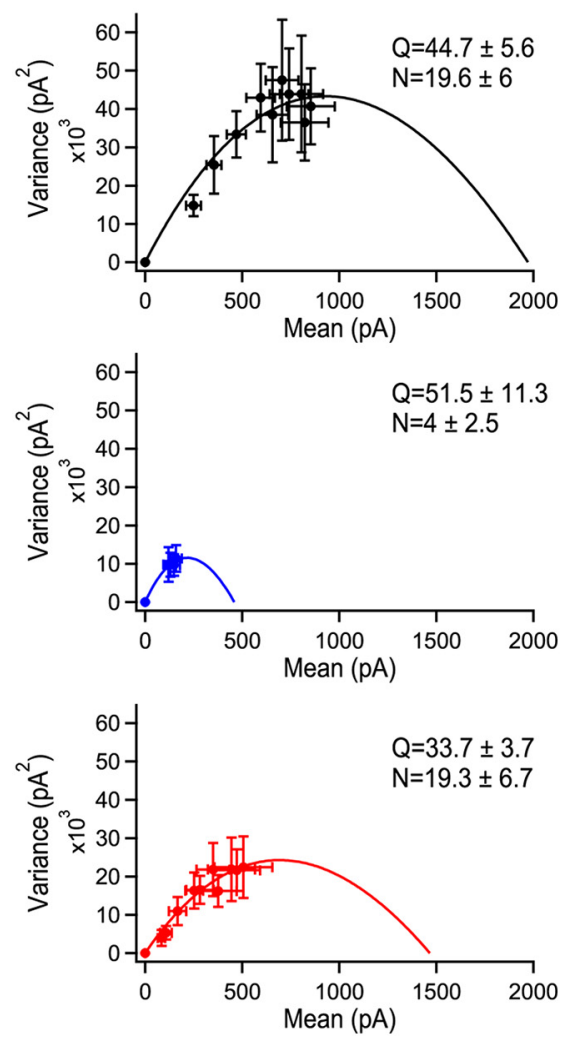

Figure 4. Varying the contribution of intrasite and intersite variability in variance-mean analysis. Simulation of the effect with the consideration that all variability in EPSC amplitudes comes from intrasite variance $(\boldsymbol{A})$, intersite variance $(\boldsymbol{B})$, or a mixed contribution of both intrasite and intersite variance $(\boldsymbol{C})$. Control is shown in black, AgTx condition in blue, and (Tx is shown in red. Note that the changes in quantal parameters caused by simulating different origins of variance is small, even across the two extreme conditions tested. The data for the AgTx conditions uses only the last four stimuli because of the changes in Q associated with the initial stimuli as calculated using covariance analysis. Data represent mean \pm SEM.

P/Q-type VGCCs can trigger the release of vesicles from more release sites, including those initially controlled by $\mathrm{N}$-type VGCCs.

\section{P/Q- and N-type VGCCs contribute distinctly to basal glutamate release}

The above results indicate that P/Q-type VGCCs have access to more release sites than $\mathrm{N}$-type VGCCs, including release sites initially controlled by $\mathrm{N}$-type under higher release probability. During trains of stimuli, the contribution of N-type VGCCs to the total EPSC amplitude is gradually decreased, highlighting the prevailing role of P/Q-type VGCCs. In these experiments, the release probability was increased by repetitively stimulating the synapse. If our conclusion holds, increasing the release probability by doubling the external calcium concentration should also decrease the contribution of N-type VGCCs. We next directly tested this hypothesis.

First, we confirmed that there was no relative change in presynaptic calcium conductance through P/Q- or N-type VGCCs when the external calcium concentration was reduced from 2.5 to $1.2 \mathrm{~mm}$. By performing presynaptic calcium imaging, we found that the relative contribution of P/Q- and N-type VGCCs to total $\mathrm{Ca}^{2+}$ influx was unchanged when recordings were performed in 1.2 or $2.5 \mathrm{~mm}$ external $\mathrm{Ca}^{2+}$ (CTx: $1.2 \mathrm{mM} \mathrm{Ca}^{2+}: 27 \pm 5.7 \%, n=$ $5 ; 2.5 \mathrm{mM} \mathrm{Ca}^{2+}: 31.5 \pm 9.3 \%, n=4 ; p>0.5$, and AgTx: $1.2 \mathrm{mM}$ $\mathrm{Ca}^{2+}: 40.3 \pm 4.4 \%, n=5 ; 2.5 \mathrm{mM} \mathrm{Ca}^{2+}: 50.5 \pm 6.9 \%, n=5$; $p>0.2$; Fig. $6 A, B)$. This indicates that the relative conductance of $\mathrm{P} / \mathrm{Q}$ - and N-type VGCCs is unaffected by a change in extracellular $\mathrm{Ca}^{2+}$.

If $\mathrm{P} / \mathrm{Q}$ - and N-type VGCCs have distinct functional roles, their relative contributions to the measured EPSC amplitude will be dependent on the external $\mathrm{Ca}^{2+}$ concentration. Given our results, this could reflect that P/Q-type VGCCs have access to more release sites or that P/Q- and N-type VGCCs are in a different coupling configuration with the calcium sensor. The contributions of P/Q- and N-type VGCCs to EPSC amplitude was similar in $1.2 \mathrm{~mm}$ external $\mathrm{Ca}^{2+}$ (AgTx: $81.2 \pm 3.5 \%$ of inhibition, $n=10$, CTx: $75.3 \pm 3.5 \%$ of inhibition, $n=10$; $p>0.2$; Fig. $6 C, D)$. Next, we increased the external $\mathrm{Ca}^{2+}$ concentration. In $2.5 \mathrm{~mm}$ external $\mathrm{Ca}^{2+}$, EPSC inhibition by CTx became significantly smaller than EPSC inhibition by AgTx (AgTx: $86.5 \pm 3.4 \%$ of inhibition, $n=14$; CTx: $61.7 \pm 3.6 \%$ of inhibition, $n=18$; $p<$ 0.001; Fig. $6 C, D$ ). Furthermore, EPSC inhibition by CTx in 2.5 $\mathrm{mm}$ external $\mathrm{Ca}^{2+}$ was also significantly smaller than in $1.2 \mathrm{~mm}$ external $\mathrm{Ca}^{2+}(p<0.05)$. Together, these data confirm that varying the external $\mathrm{Ca}^{2+}$ concentration changes the relative contribution of P/Q- and N-type VGCCs to EPSC amplitude. Therefore, together with the above conclusions, these results argue that $\mathrm{P} / \mathrm{Q}$-type VGCCs have access to more release sites and that P/Q- and N-type VGCCs are coupled differently to the calcium sensor. 
A

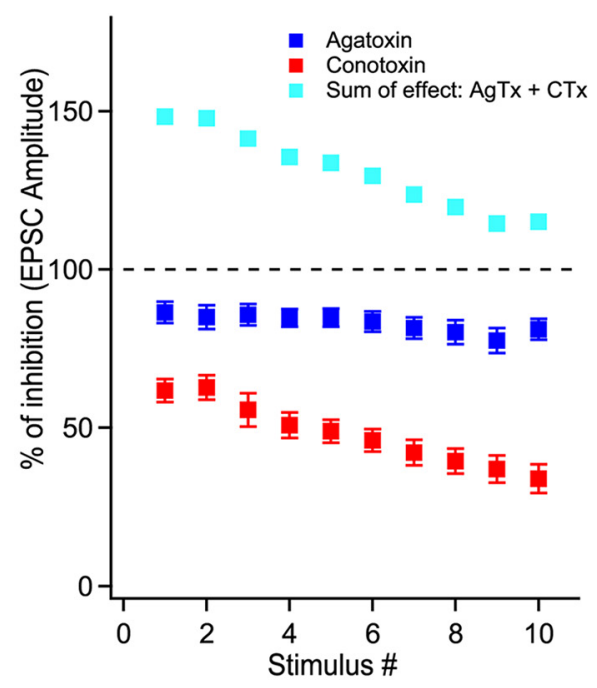

D

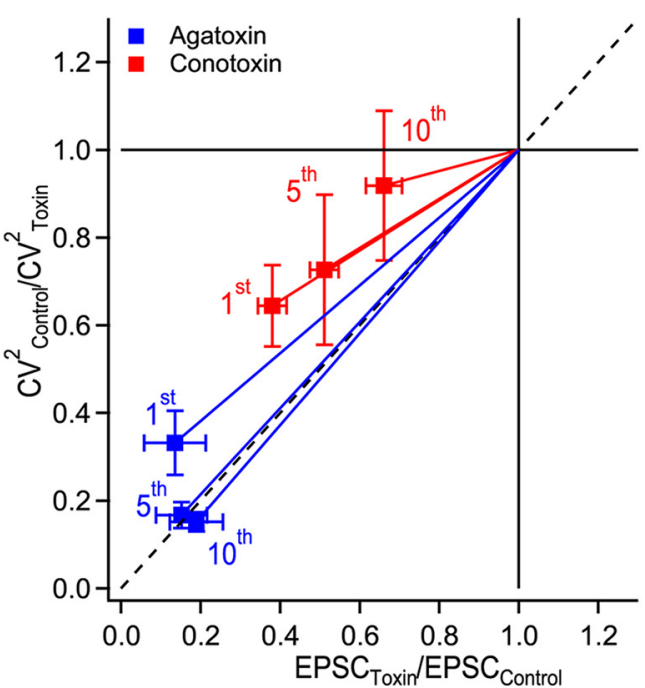

B

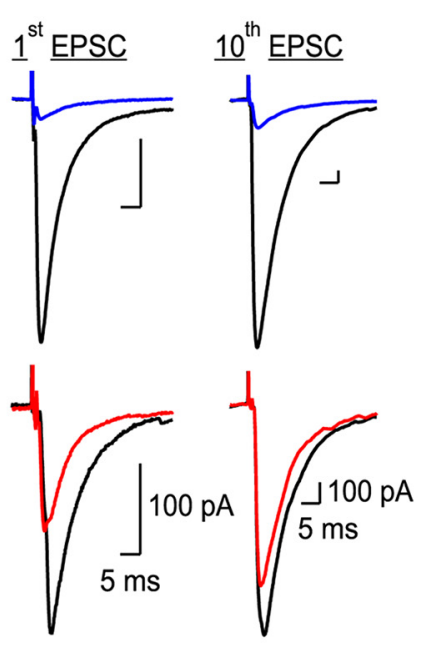

E1

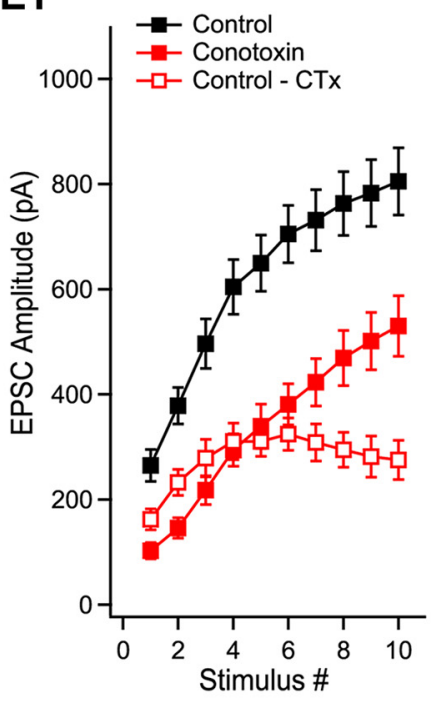

C

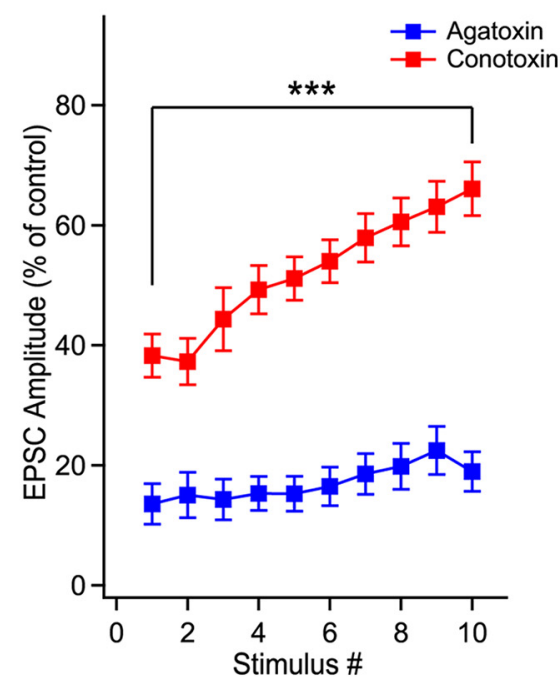

E2

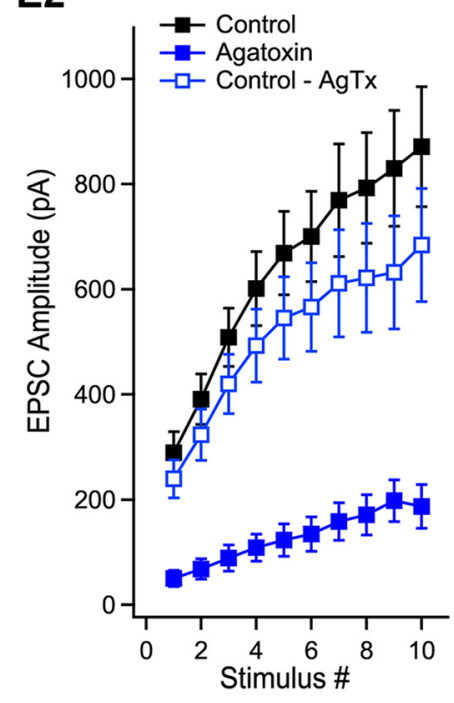

Figure 5. P/Q-type VGCCs contribute to glutamate release at active zones initially controlled by N-type VGCCs. A, Supra-additive effect of AgTx and CTx is gradually reduced when the release probability is increased during trains. The percentage of EPSC amplitude inhibition as a function of stimulus number for AgTx (blue), (Tx (red), and their arithmetic sum (turquoise). The arithmetic sum initially shows large supra-additivity that is reduced with increase in release probability. $\boldsymbol{B}$, Representative examples of MF-evoked EPSCs in control (black) and in the presence of AgTx (blue) or CTx (red) for the 1st and 10th stimuli in the train. C, The percentage of effect of blocking N-type VGCCs on EPSC amplitude is gradually decreased during EPSC trains. D, CV analysis of EPSCS reveals that P/Q- but not N-types VGCCs are essential to recruit additional release sites later during trains of facilitating EPSCs. Data points from the 1st, 5 th, and 10th stimuli in AgTx are found close to the diagonal. This indicates that AgTx decreases the number of active release sites for all stimuli in the train. In contrast, data points in CTx shift toward the horizontal line for the last stimuli. This suggests that N-type VGCCs control the quantal size toward the end of the train, but not the number of active release sites. E1, Subtracting the EPSC size measured in CTx from control reveals the contribution of N-type VGCCs to EPSC amplitude. A plateau portion is reached after the first five stimuli. E2, The contribution of P/Q-type VGCCs to EPSC amplitude, as revealed by subtracting the EPSC size recorded in AgTx, increases during EPSC trains. Data represent mean \pm SEM. ${ }^{* * *} p<0.001$.

\section{P/Q-type VGCCs leverage microdomain signaling to recruit} additional release sites

Our results show that P/Q- and N-type VGCCs control specific $\mathrm{Ca}^{2+}$ spatiotemporal dynamics in MF terminals, with $\mathrm{P} / \mathrm{Q}$-type VGCCs gating spatially homogeneous $\mathrm{Ca}^{2+}$ elevations (Fig. 1). As a result, N-type VGCCs support short-term facilitation through synchronization of multivesicular release, whereas P/Qtype VGCCs are essential for the recruitment of additional release sites (Figs. 2-4). Furthermore, P/Q-type VGCCs have access to multiple active zones, whereas $\mathrm{N}$-type VGCCs are restricted to a portion of release sites (Fig. 5, 6). Based on this evidence, we hypothesized that N-type VGCCs are tightly coupled to the $\mathrm{Ca}^{2+}$ sensor at a limited number of release sites, whereas P/Q-type VGCCs trigger release through microdomain signaling.
To test this hypothesis, we first performed experiments under condition of $1.2 \mathrm{~mm}$ external $\mathrm{Ca}^{2+}$ and applied AgTx, CTx, or the membrane-permeant slow calcium chelator EGTA-AM. EGTA largely decreases release mediated through microdomain signaling with no effect on release mediated by nanodomain signaling. Application of EGTA-AM $(100 \mu \mathrm{M})$ on trains of EPSCs decreased the amplitude of all EPSCs in the train (Fig. $7 A-C$ ), but did not fully block short-term facilitation (Fig. 7A-C). Strikingly, EGTA-AM reduced short-term facilitation more than CTx ( $p<0.05$ at the 10th stimulus; $n=8$ for EGTA-AM; $n=11$ for CTx), and to a level similar as $\operatorname{AgTx}(p>0.25$ at the 10th stimulus; $n=10$ for AgTx; Fig. 7C). Next, to verify whether the effect of EGTA-AM and AgTx were also similar throughout the trains, we normalized EPSC amplitudes to control at their respective posi- 

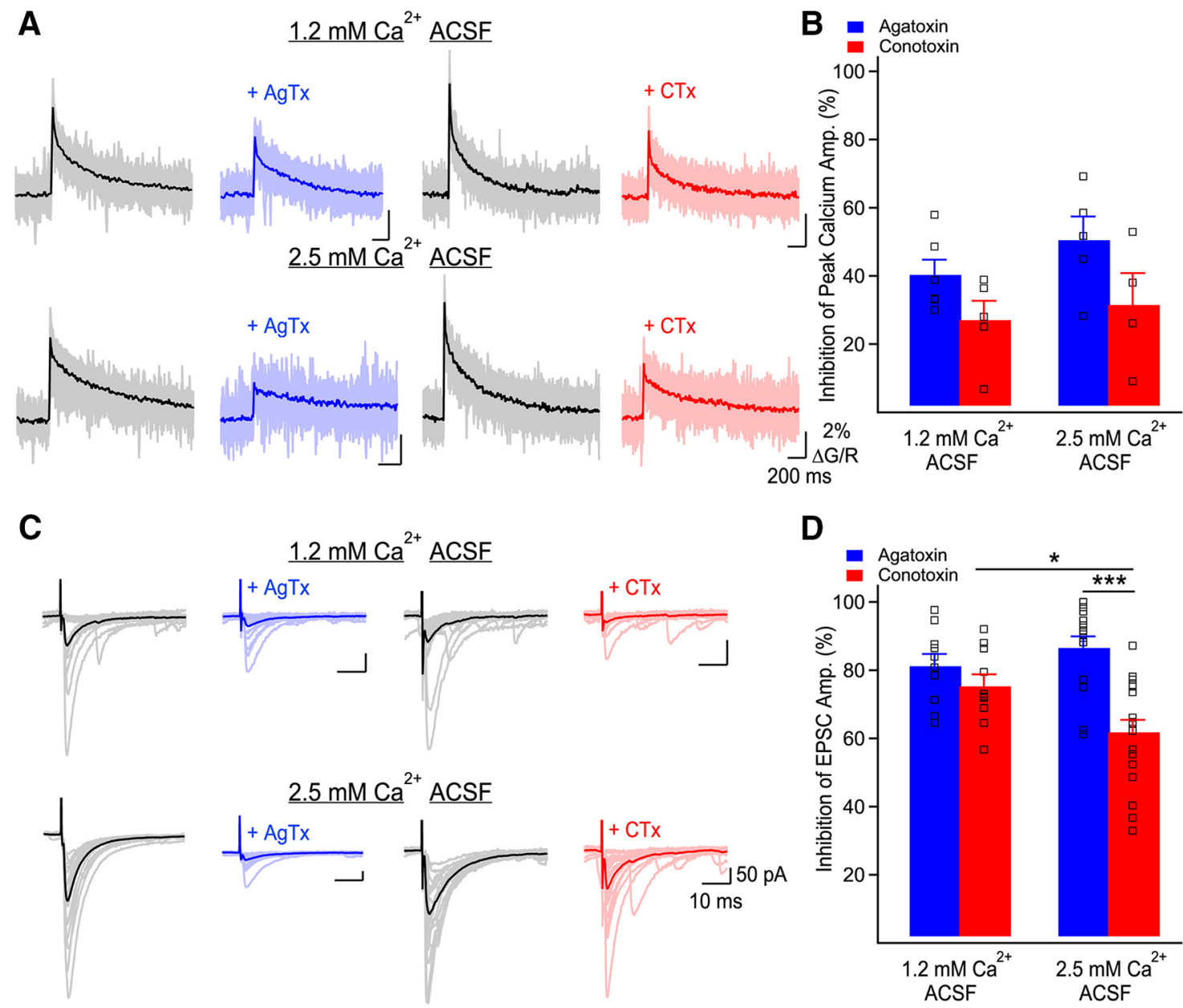

Figure 6. $\mathrm{Ca}^{2+}$ influx through $\mathrm{P} / \mathrm{Q}$ - and N-type VGCCs differently control basal glutamate release. $A$, Representative examples of $\mathrm{Ca}^{2+}$ elevations recorded in MF boutons using Fluo-5F before and following application of $\mathrm{AgTx}$ or $\mathrm{CTx}$, in both external $\mathrm{Ca}^{2+}$ concentrations. Individual traces correspond to individual points recorded simultaneously in MF boutons with the average overlaid. Traces are the average of 20 trials. B, Bar graph showing the inhibition of peak $\mathrm{Ca}^{2+}$ transients' amplitude by AgTx and CTx as a function of the external $\mathrm{Ca}^{2+}$ concentration. The relative contribution of P/Q- and N-type VGCCs to bouton-averaged $\mathrm{Ca}^{2+}$ elevations is not affected by the external $\mathrm{Ca}^{2+}$ concentration. Squares show data from individual boutons. C, Representative examples of MF-evoked EPSCs recorded in CA3 pyramidal cells in control conditions and in the presence of AgTx (blue) or CTx (red). The external calcium concentration was set to 1.2 or $2.5 \mathrm{~mm}$. Traces shown are 20 consecutive trials and their average. $\boldsymbol{D}$, Bar graph showing the percentage of EPSC inhibition by AgTx and CTx as a function of the external $\mathrm{Ca}^{2+}$ concentration. Squares show individual neurons. Data represent mean \pm SEM. ${ }^{*} p<0.05,{ }^{* * *} p<0.001$.

tions (stimuli 1-10) during the trains (Fig. 7D). We observed that EGTA-AM and AgTx had a similar effect on the EPSC amplitude, reducing EPSCs by a larger fraction later during the trains. This effect was in contrast to the effect of CTx, which blocked EPSC by a lesser fraction later during the trains (Fig. 7D). In addition, the effect of AgTx on EPSCs was slightly larger than the effect of EGTA-AM for every EPSC (Fig. $7 B-D$ ). However, the time course of the effect of EGTA-AM and AgTx were identical (Fig. $7 E$ ). These data indicate that disrupting microdomain calcium signaling with EGTA-AM produces an identical effect on shortterm facilitation as blocking P/Q-type VGCCs.

We next investigated whether the alteration of short-term facilitation caused by EGTA-AM and AgTx are mediated through a similar mechanism using CV analysis. Short-term facilitation following EGTA-AM or AgTx application occurred exclusively through the synchronization of multivesicular release, as the data points fell on the horizontal line (Fig. $7 F$ ). This effect of EGTA-AM and AgTx were in contrast to the effect of CTx, which could not abolish the recruitment of additional release sites (Fig. $7 F$ ). AgTx prevents the recruitment of additional release sites in a way almost identical to EGTA-AM. Therefore, these results indi- cate that P/Q-type VGCCs operate mainly through microdomain signaling.

Next, to evaluate the contribution of loosely coupled P/Q- and tightly coupled N-type VGCCs to EPSC amplitude, we sequentially applied EGTA-AM and AgTx or CTx. We measured the fraction of EPSCs blocked by AgTx in presence of EGTA-AM to quantify release mediated exclusively by tightly coupled $\mathrm{P} / \mathrm{Q}$-type VGCCs (Fig. 8A1, A2). The full contribution of P/Q-type VGCCs was evaluated by EPSCs generated in CTx, in absence of EGTAAM. We found that the tightly coupled fraction of P/Q-type VGCCs could not explain the full contribution of $\mathrm{P} / \mathrm{Q}$-type VGCCs later during the train (Fig. 8A2), indicative of loose coupling. In sharp contrast, the tightly coupled fraction of N-types VGCCs was the same as the full contribution of N-type VGCCs (Fig. 8B2). This is well demonstrated by the almost perfect overlap of the two curves (Fig. 8B2). Therefore, these results confirm that P/Q-type VGCCs act through loose and tight coupling, whereas N-type VGCCs are exclusively tightly coupled to calcium sensors.

We observed that when the release probability is elevated, $\mathrm{P} / \mathrm{Q}$-type VGCCs recruit additional release sites. When P/Q-type 
A
$1.2 \mathrm{mM} \mathrm{Ca}^{2+} \underline{\mathrm{ACSF}}$

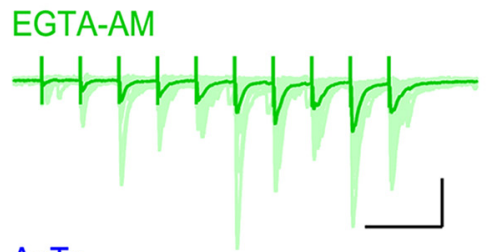

$\operatorname{AgTx}$

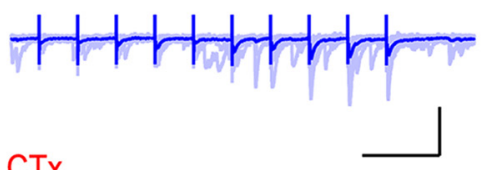

CTx

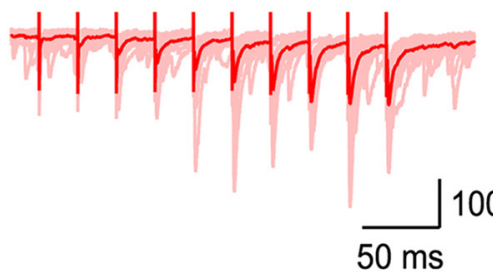

B

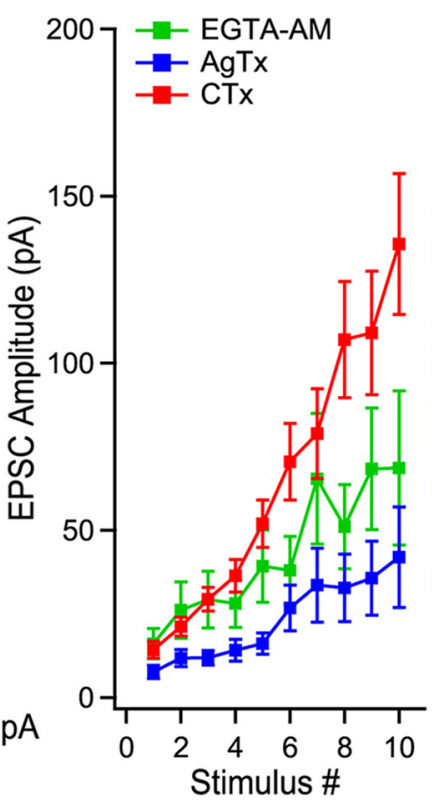

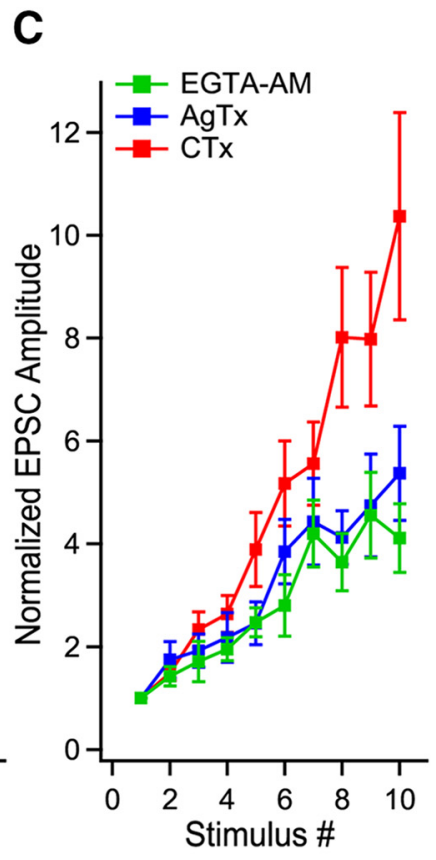

D

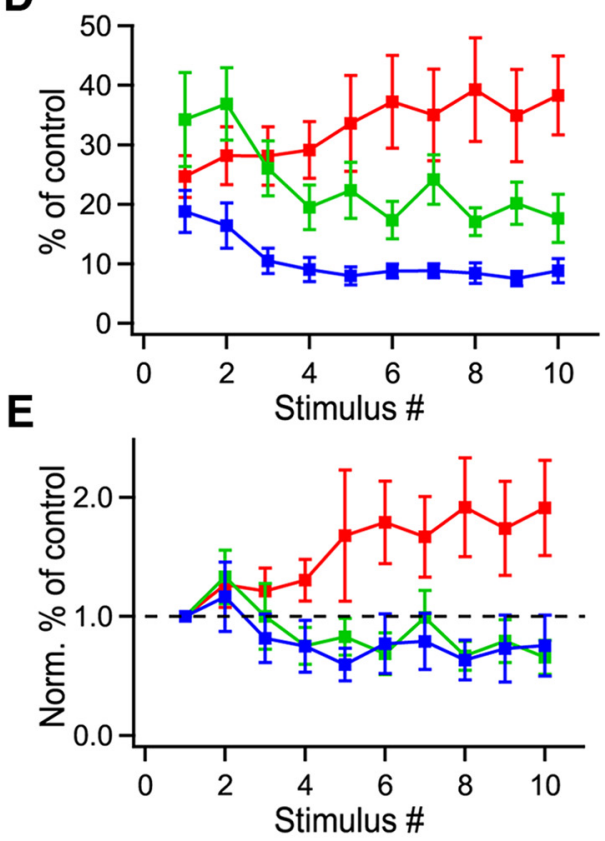

Figure 7. P/Q-type VGCCs recruit additional release sites through microdomain signaling. $A$, MF-evoked EPSCs recorded presence of EGTA-AM (green), AgTx (blue), or CTx (red) in the condition of $1.2 \mathrm{~mm} \mathrm{Ca}^{2+}$ ACSF. B, EPSC amplitude as a function of stimulus number in the presence of EGTA-AM (green; $n=8$ ), AgTx (blue; $n=10$ ) or CTx (red; $n=11$ ). C, Normalized EPSC amplitude as a function of stimulus number showing the identical progression of EPSC amplitude in the presence of EGTA-AM or AgTx. D, Normalized EPSC amplitude to their respective position in control conditions during trains, as a function of stimulus number. $\boldsymbol{E}$, Data shown in $\boldsymbol{D}$, normalized to the first EPSC in the train to show the time course of effect of EGTA-AM, AgTx, and CTx. Although EGTA-AM and AgTx have a quasi-identical effect, EPSCs mediated in the presence of (Tx are gradually enhanced during trains. $\boldsymbol{F}$, CV analysis shows that EGTA-AM (green; $n=8$ ) blocks the recruitment of additional release sites like AgTx (blue; $n=10$ ). However, CTx (red; $n=11$ ) could not block the recruitment of additional release sites. Data represent mean \pm SEM.

VGCCs were the sole contributors to short-term facilitation (in the presence of CTx), the inhibition of EPSCs was larger in the beginning than in the end of the train (Figs. $5 A-C, 7 D, E$ ). Therefore, coapplication of CTx and EGTA-AM should confirm the microdomain coupling of P/Q-type VGCCs by reversing this effect. Indeed, our results show that the EPSC percentage of control was now significantly reduced when in CTx + EGTA-AM ( $p<0.01$; Fig. $8 C)$, instead of being increased. Altogether, these results are fully consistent with the fact that
P/Q-type VGCCs promote release mainly through microdomain but also nanodomain signaling, whereas N-type VGCCs act through nanodomain signaling.

\section{Discussion}

Our findings identify how P/Q- and N-type VGCCs differently shape the $\mathrm{Ca}^{2+}$ landscape in MF terminals to release glutamate. Whereas P/Q-type VGCCs generate spatially homogeneous $\mathrm{Ca}^{2+}$ elevations, N-type VGCCs support highly heterogeneous 
A1

Control

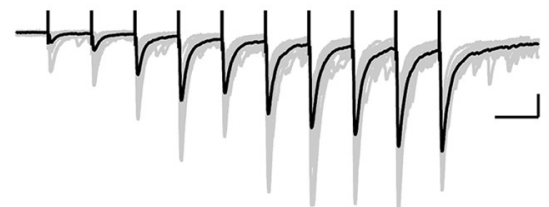

AgTx + EGTA-AM

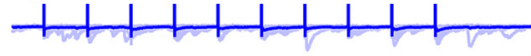

A2

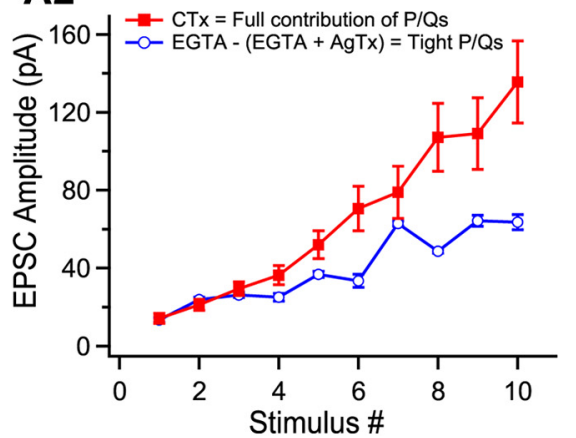

B1

Control

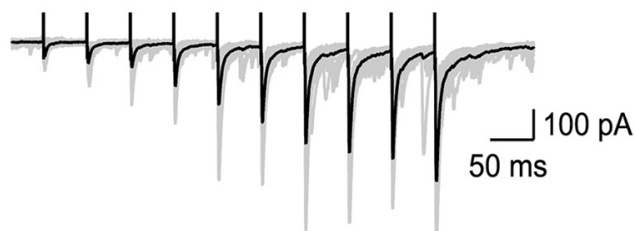

CTx + EGTA-AM

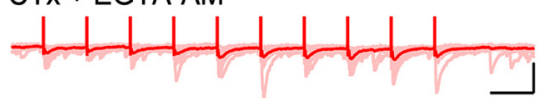

B2

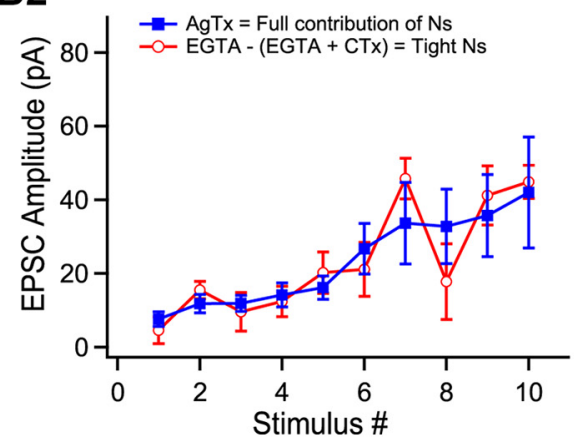

C

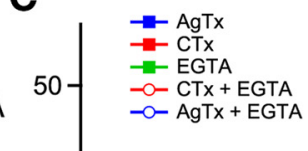

Figure 8. N-type VGCCs act exclusively through nanodomain signaling. A1, Representative examples of EPSCS recorded in control (black) and in the presence of AgTx + EGTA-AM (blue). A2, EPSC amplitude of tightly coupled P/Q-type VGCCs (open blue circles) compared with the EPSC amplitude generated by the total population of P/Q-type VGCCs (red square). B1, Example of EPSCs recorded in control condition (black) and following the application of (Tx + EGTA-AM (red). B2, Graph showing the EPSC amplitude generated by tightly coupled N-type VGCCs (open red circles) compared with the EPSC amplitude evoked by all N-type VGCCs during trains of stimuli. C, Percentage of effect of toxins, EGTA-AM, and combined application of compounds on the 1st and 10th EPSCs generated in trains. Coapplication of CTx and EGTA-AM prevents the decrease in the percentage of effect of CTx during trains. Data represent mean \pm SEM. ${ }^{* *} p<0.01$.

$\mathrm{Ca}^{2+}$ transients. The distinct spatial profiles of $\mathrm{Ca}^{2+}$ elevations generated by $\mathrm{P} / \mathrm{Q}$ - or N-type VGCCs support their functional role. Indeed, $\mathrm{Ca}^{2+}$ entering through P/Q-type VGCCs control a large number of release sites, act through microdomain $\mathrm{Ca}^{2+}$ signaling, and control short-term facilitation by recruiting additional release sites. In contrast, N-type VGCCs mediate release through nanodomain coupling at a limited number of release sites and support short-term facilitation by enhancing multivesicular release. MF terminals are endowed with a large number of active zones, possess a large pool of vesicles, and exhibit notorious short-term facilitation (Toth et al., 2000; Urban et al., 2001; Rollenhagen et al., 2007). Therefore, the segregation of functions between P/Q- and N-type VGCCs observed here optimizes the use of the presynaptic machinery available to trigger an AP in the postsynaptic CA3 pyramidal cell during repetitive granule cell firing, their preferred firing mode (Csicsvari et al., 2003; Pernía-Andrade and Jonas, 2014).

Two calcium source to sensor coupling configurations coexist in MF terminals

In behaving animals, granule cells act as low-pass filters to transform the dense cortical code into a sparse hippocampal coding. Granule cells fire high-frequency APs, leading to $\mathrm{Ca}^{2+}$-dependent short-term facilitation of glutamate release (Salin et al., 1996; Klausnitzer and Manahan-Vaughan, 2008). Our results show that tightly coupled N-type VGCCs are well positioned to support the synchronization of multivesicular release, whereas loosely coupled P/Q-type VGCCs contribute to the recruitment of additional release sites. This presynaptic configuration of VGCCs is in sharp contrast to the juvenile calyx of Held, where P/Q-type VGCCs are tightly coupled to the $\mathrm{Ca}^{2+}$ sensor and N-type VGCCs are loosely coupled (Iwasaki et al., 2000). However, in adults this segregation disappears, and the full $\mathrm{Ca}^{2+}$ influx is mediated by tightly coupled P/Q-type VGCCs. Comparatively, the calyx of Held is composed of several hundred release sites but exhibits short-term depression upon repetitive activation (Wu et al., 1999). The disposition of VGCCs is therefore of crucial importance to control short-term dynamics of neurotransmitter release. What is the advantage of combining two coupling configurations? We propose that this dual coupling configuration allows the integration of activity over a broad range of AP frequency and number during burst, which are very variable in granule cells (Pernía-Andrade and Jonas, 2014).

\section{Spatiotemporal dynamics of $\mathrm{Ca}^{2+}$ elevations in giant MF terminals}

Spatially heterogeneous $\mathrm{Ca}^{2+}$ elevations in MF terminals are generated through P/Q- and N-type VGCCs. Interestingly, P/Q-type VGCCs support more homogeneous $\mathrm{Ca}^{2+}$ elevations than $\mathrm{N}$-type VGCCs. The spatiotemporal dynamics of $\mathrm{Ca}^{2+}$ and interactions with endogenous buffers are thought to underlie short-term facilitation at this synapse. Following $\mathrm{Ca}^{2+}$ entry in the presynaptic terminal, $\mathrm{Ca}^{2+}$ ions are rapidly bound to endogenous calcium buffers. Calcium elevations in MF boutons are atypically long-lasting, with a slow calcium extrusion rate compared with other small presynaptic terminals (Scott and Rusakov, 2006; Ermolyuk et al., 2013). Endogenous $\mathrm{Ca}^{2+}$ buffer in MF boutons have fast kinetics, a concentration of $100-300 \mu \mathrm{M}$, demonstrate high-affinity, and are highly mobile (Vyleta and Jonas, 2014). Also, following a single AP, Vyleta and Jonas observed a global saturation of the endogenous calcium buffers. How can endogenous $\mathrm{Ca}^{2+}$ buffers support intraterminal distribution of $\mathrm{Ca}^{2+}$ and allow for specific neurotransmitter release properties? A recent study investigating $\mathrm{Ca}^{2+}$ buffering in cerebellar MF boutons revealed that fixed endogenous $\mathrm{Ca}^{2+}$ buffer had a lower affinity 
than mobile $\mathrm{Ca}^{2+}$ buffers, enabling fast $\mathrm{Ca}^{2+}$ clearance from active zones (Delvendahl et al., 2015). This research provided direct evidence for regions with different buffering properties in presynaptic terminals. It is therefore a possibility that P/Q- and $\mathrm{N}$-type VGCCs could have different association with the endogenous calcium buffers in hippocampal MF boutons.

We used random-access two-photon calcium imaging to track the spatial dynamics of calcium elevations in giant MF boutons. This approach offers the advantage of providing both a high temporal and spatial resolution, but there is a tradeoff between both parameters. Indeed, under such an imaging paradigm, the spatial resolution is inversely related to the temporal resolution. We optimized both parameters by placing a limited number of points $(<54)$ on the full surface of the boutons, yielding a temporal resolution which still allowed to precisely determine the peak calcium transient. Two important limitations for the spatial and temporal resolution should be considered. First, our measurements are diffraction-limited, with the spatial resolution determined by the resolution of the microscope (see Materials and Methods). As a result, recorded calcium elevations correspond to microdomains. Therefore, our findings with calcium imaging cannot be extrapolated to the calcium nanodomains. Second, a sufficient temporal resolution is mandatory to determine the peak amplitude of the calcium transients with precision. Given that the addition of $385 \mu \mathrm{M}$ Fluo-5F significantly slows down the calcium transients, the temporal resolution used here is sufficient to obtain a satisfactory measurement of the peak amplitude.

\section{Functional specialization of P/Q- and N-type VGCCs control short-term facilitation}

How do specialized $\mathrm{Ca}^{2+}$ spatiotemporal dynamics from $\mathrm{N}$ - and $\mathrm{P} / \mathrm{Q}$-type VGCCs in MF terminals favor short-term facilitation? Stochastic activation of VGCCs is clearly insufficient to control short-term facilitation in a graded way, because VGCCs in MF boutons demonstrate a high open probability of $72 \%$ following single APs (Bischofberger et al., 2002). Therefore, other mechanisms must support short-term facilitation through the two coupling configurations observed.

How do tightly coupled N-type VGCCs support short-term facilitation through multivesicular release? Three mechanisms could contribute. First, it was demonstrated through single-channel tracking that VGCCs are highly mobile in presynaptic terminal membranes (Mercer et al., 2011; Schneider et al., 2015). It is possible that the distance between N-type VGCCs and vesicles decreases in an activity-dependent manner, thereby facilitating vesicle release. Second, at CA3-CA1 pyramidal cell synapses, Cav2.2 channels experience voltage-dependent tonic inhibition by $\mathrm{G}_{\mathrm{o} / i}$, which facilitate $\mathrm{Ca}^{2+}$ currents during repetitive activity (Scheuber et al., 2004). Therefore, activity-dependent augmentation in the $\mathrm{Ca}^{2+}$ current through N-type VGCCs could enhance multivesicular release. Third, in large MF boutons, N-type VGCCs are activated less efficiently than $\mathrm{P} / \mathrm{Q}$-type channels, but AP broadening during trains of activity can increase their contribution significantly (Li et al., 2007). At MF-CA3 synapses, significant broadening of the AP occurs during trains of APs (Geiger and Jonas, 2000), which could contribute to boosting $\mathrm{Ca}^{2+}$ currents through N-type VGCCs. Therefore, these activity-dependent mechanisms could be key in ensuring that release sites fully operate through multivesicular release during repetitive activity.

Interestingly, $\mathrm{Ca}^{2+}$ entering through $\mathrm{N}$-type VGCCs is less important than $\mathrm{Ca}^{2+}$ from P/Q-type VGCCs for short-term facilitation. This is consistent with microdomain signaling by P/Q-type VGCCs, which have access to more release sites. These results are crucial given the recently demonstrated key role of endogenous buffer saturation for short-term facilitation at MFCA3 synapses (Vyleta and Jonas, 2014). Indeed, the calcium source to sensor distance was found to be $70-88 \mathrm{~nm}$, longer than the 10-30 nm typically found in typical CNS synaptic terminals (Eggermann et al., 2012; Vyleta and Jonas, 2014). This relatively long distance permits endogenous $\mathrm{Ca}^{2+}$ buffer to compete for $\mathrm{Ca}^{2+}$ ions with sensors triggering vesicle release and conducts to short-term facilitation by buffer saturation during repetitive activity (Jackson and Redman, 2003; Matveev et al., 2004). The results presented here are in full agreement that loose-coupling is the favored coupling mode to mediate short-term facilitation at the MF-CA3 pyramidal cell synapses.

At several synapses, VGCCs can be found outside active zones (Wu et al., 1999). What is the role of these VGCCs? We demonstrate that homogeneous $\mathrm{Ca}^{2+}$ elevations in MF terminals through loosely coupled P/Q-type VGCCs are essential for the recruitment of additional release sites. How are these release sites controlled? Our findings support the idea that expression of VGCCs is not homogeneous at a single release site, with no release sites sensing $\mathrm{Ca}^{2+}$ exclusively from either P/Q- or N-type VGCCs. Functional analysis demonstrate that only a few release sites have access to $\mathrm{Ca}^{2+}$ entering from N-type VGCCs. These release sites have a higher release probability. The large number of release sites at the MF synapse allows the synapse to release neurotransmitter in a graded way, with lower release probability release sites recruited later during sustained activity (Chamberland et al., 2014; Stanley, 2015). This allows MF terminals to overcome the physical limitations imposed by the number of available release sites and optimize the use of a large vesicle pool (Neher, 2010).

Together, our results suggest that functional specialization of VGCCs support the physiological function of the synapse. At MF terminals, P/Q-type VGCCs control a larger number of release sites to support short-term facilitation through microdomain coupling. N-type VGCCs operate through nanodomain coupling at a few release sites and tune multivesicular release. We find that $\mathrm{P} / \mathrm{Q}$ - and N-type VGCCs have segregated physiological functions because of the spatiotemporal dynamics of $\mathrm{Ca}^{2+}$ they generate, rather than specific biophysical properties. Therefore, the functional specialization of P/Q- and N-type VGCCs optimizes the use of the large number of release sites and the large pool of vesicles found in giant MF terminals.

\section{References}

Amaral DG, Dent JA (1981) Development of the mossy fibers of the dentate gyrus: I. A light and electron microscopic study of the mossy fibers and their expansions. J Comp Neurol 195:51-86. CrossRef Medline

Bekkers JM, Stevens CF (1990) Presynaptic mechanism for long-term potentiation in the hippocampus. Nature 346:724-729. CrossRef Medline

Bertram R, Sherman A, Stanley EF (1996) Single-domain/bound calcium hypothesis of transmitter release and facilitation. J Neurophysiol 75: 1919-1931. Medline

Bischofberger J, Geiger JR, Jonas P (2002) Timing and efficacy of Ca2+ channel activation in hippocampal mossy fiber boutons. J Neurosci 22: 10593-10602. Medline

Borst JG, Sakmann B (1996) Calcium influx and transmitter release in a fast CNS synapse. Nature 383:431-434. CrossRef Medline

Castillo PE, Weisskopf MG, Nicoll RA (1994) The role of Ca2 + channels in hippocampal mossy fiber synaptic transmission and long-term potentiation. Neuron 12:261-269. CrossRef Medline

Chamberland S, Evstratova A, TóthK (2014) Interplay between synchronization of multivesicular release and recruitment of additional release sites support short-term facilitation at hippocampal mossy fiber to CA3 pyramidal cells synapses. J Neurosci 34:11032-11047. CrossRef Medline

Clements JD, Silver RA (2000) Unveiling synaptic plasticity: a new graphical and analytical approach. Trends Neurosci 23:105-113. CrossRef Medline 
Csicsvari J, Jamieson B, Wise KD, Buzsáki G (2003) Mechanisms of gamma oscillations in the hippocampus of the behaving rat. Neuron 37:311-322. CrossRef Medline

Delvendahl I, Jablonski L, Baade C, Matveev V, Neher E, Hallermann S (2015) Reduced endogenous Ca2 + buffering speeds active zone Ca2+ signaling. Proc Natl Acad Sci U S A 112:E3075-3084. CrossRef Medline

Dietrich D, Kirschstein T, Kukley M, Pereverzev A, von der Brelie C, Schneider T, Beck H (2003) Functional specialization of presynaptic Cav2.3 Ca2 + channels. Neuron 39:483-496. CrossRef Medline

Eggermann E, Bucurenciu I, Goswami SP, Jonas P (2012) Nanodomain coupling between $\mathrm{Ca}(2)(+)$ channels and sensors of exocytosis at fast mammalian synapses. Nat Rev Neurosci 13:7-21. CrossRef Medline

Ermolyuk YS, Alder FG, Surges R, Pavlov IY, Timofeeva Y, Kullmann DM, Volynski KE (2013) Differential triggering of spontaneous glutamate release by P/Q-, N- and R-type Ca2+ channels. Nat Neurosci 16:17541763. CrossRef Medline

Faber DS, Korn H (1991) Applicability of the coefficient of variation method for analyzing synaptic plasticity. Biophys J 60:1288-1294. CrossRef Medline

Frerking M, Wilson M (1996) Effects of variance in mini amplitude on stimulus-evoked release: a comparison of two models. Biophys J 70:2078 2091. CrossRef Medline

Geiger JR, Jonas P (2000) Dynamic control of presynaptic Ca(2+) inflow by fast-inactivating $\mathrm{K}(+)$ channels in hippocampal mossy fiber boutons. Neuron 28:927-939. CrossRef Medline

Henze DA, Wittner L, Buzsáki G (2002) Single granule cells reliably discharge targets in the hippocampal CA3 network in vivo. Nat Neurosci 5:790-795. Medline

Ishikawa T, Kaneko M, Shin HS, Takahashi T (2005) Presynaptic N-type and $\mathrm{P} / \mathrm{Q}$-type $\mathrm{Ca} 2+$ channels mediating synaptic transmission at the calyx of Held of mice. J Physiol 568:199-209. CrossRef Medline

Iwasaki S, Momiyama A, Uchitel OD, Takahashi T (2000) Developmental changes in calcium channel types mediating central synaptic transmission. J Neurosci 20:59-65. Medline

Jackson MB, Redman SJ (2003) Calcium dynamics, buffering, and buffer saturation in the boutons of dentate granule-cell axons in the hilus. J Neurosci 23:1612-1621. Medline

Jung MW, McNaughton BL (1993) Spatial selectivity of unit activity in the hippocampal granular layer. Hippocampus 3:165-182. CrossRef Medline

Klausnitzer J, Manahan-Vaughan D (2008) Frequency facilitation at mossy fiber-CA3 synapses of freely behaving rats is regulated by adenosine A1 receptors. J Neurosci 28:4836-4840. CrossRef Medline

Larkman AU, Jack JJ, Stratford KJ (1997) Quantal analysis of excitatory synapses in rat hippocampal CAl in vitro during low-frequency depression. J Physiol 505:457-471. CrossRef Medline

Li L, Bischofberger J, Jonas P (2007) Differential gating and recruitment of P/Q-, N-, and R-type Ca2+ channels in hippocampal mossy fiber boutons. J Neurosci 27:13420-13429. CrossRef Medline

Luebke JI, Dunlap K, Turner TJ (1993) Multiple calcium channel types control glutamatergic synaptic transmission in the hippocampus. Neuron 11:895-902. CrossRef Medline

Malinow R, Tsien RW (1990) Presynaptic enhancement shown by wholecell recordings of long-term potentiation in hippocampal slices. Nature 346:177-180. CrossRef Medline

Matveev V, Zucker RS, Sherman A (2004) Facilitation through buffer saturation: constraints on endogenous buffering properties. Biophys J 86: 2691-2709. CrossRef Medline

Mercer AJ, Chen M, Thoreson WB (2011) Lateral mobility of presynaptic L-type calcium channels at photoreceptor ribbon synapses. J Neurosci 31:4397-4406. CrossRef Medline

Meyer AC, Neher E, Schneggenburger R (2001) Estimation of quantal size and number of functional active zones at the calyx of Held synapse by nonstationary EPSC variance analysis. J Neurosci 21:7889-7900. Medline

Mintz IM, Sabatini BL, Regehr WG (1995) Calcium control of transmitter release at a cerebellar synapse. Neuron 15:675-688. CrossRef Medline

Neher E (2010) What is rate-limiting during sustained synaptic activity: vesicle supply or the availability of release sites. Front Synaptic Neurosci 2:144. CrossRef Medline

Nusser Z (2009) Variability in the subcellular distribution of ion channels increases neuronal diversity. Trends Neurosci 32:267-274. CrossRef Medline

Otsu Y, Bormuth V, Wong J, Mathieu B, Dugué GP, Feltz A, Dieudonné S (2008) Optical monitoring of neuronal activity at high frame rate with a digital random-access multiphoton (RAMP) microscope. J Neurosci Methods 173:259-270. CrossRef Medline

Pelkey KA, Topolnik L, Lacaille JC, McBain CJ (2006) Compartmentalized $\mathrm{Ca}(2+)$ channel regulation at divergent mossy-fiber release sites underlies target cell-dependent plasticity. Neuron 52:497-510. CrossRef Medline

Pernía-Andrade AJ, Jonas P (2014) Theta-gamma-modulated synaptic currents in hippocampal granule cells in vivo define a mechanism for network oscillations. Neuron 81:140-152. CrossRef Medline

Reid CA, Bekkers JM, Clements JD (2003) Presynaptic Ca2+ channels: a functional patchwork. Trends Neurosci 26:683-687. CrossRef Medline

Rollenhagen A, Sätzler K, Rodríguez EP, Jonas P, Frotscher M, Lübke JH (2007) Structural determinants of transmission at large hippocampal mossy fiber synapses. J Neurosci 27:10434-10444. CrossRef Medline

Salin PA, Scanziani M, Malenka RC, Nicoll RA (1996) Distinct short-term plasticity at two excitatory synapses in the hippocampus. Proc Natl Acad Sci U S A 93:13304-13309. CrossRef Medline

Scheuber A, Miles R, Poncer JC (2004) Presynaptic Cav2.1 and Cav2.2 differentially influence release dynamics at hippocampal excitatory synapses. J Neurosci 24:10402-10409. CrossRef Medline

Scheuss V, Schneggenburger R, Neher E (2002) Separation of presynaptic and postsynaptic contributions to depression by covariance analysis of successive EPSCs at the calyx of Held synapse. J Neurosci 22:728-739. Medline

Schneider R, Hosy E, Kohl J, Klueva J, Choquet D, Thomas U, Voigt A, Heine M (2015) Mobility of calcium channels in the presynaptic membrane. Neuron 86:672-679. CrossRef Medline

Scimemi A, Diamond JS (2012) The number and organization of Ca2+ channels in the active zone shapes neurotransmitter release from Schaffer collateral synapses. J Neurosci 32:18157-18176. CrossRef Medline

Scott R, Rusakov DA (2006) Main determinants of presynaptic Ca2+dynamics at individual mossy fiber-CA3 pyramidal cell synapses. J Neurosci 26:7071-7081. CrossRef Medline

Silver RA (2003) Estimation of nonuniform quantal parameters with multiple-probability fluctuation analysis: theory, application and limitations. J Neurosci Methods 130:127-141. CrossRef Medline

Simon SM, Llinás RR (1985) Compartmentalization of the submembrane calcium activity during calcium influx and its significance in transmitter release. Biophys J 48:485-498. CrossRef Medline

Stanley EF (2015) Single calcium channel domain gating of synaptic vesicle fusion at fast synapses; analysis by graphic modeling. Channels 9:324333. CrossRef Medline

Takahashi T, Momiyama A (1993) Different types of calcium channels mediate central synaptic transmission. Nature 366:156-158. CrossRef Medline

Toth K, Suares G, Lawrence JJ, Philips-Tansey E, McBain CJ (2000) Differential mechanisms of transmission at three types of mossy fiber synapse. J Neurosci 20:8279-8289. Medline

Tringham EW, Dupere JR, Payne CE, Usowicz MM (2008) Protease treatment of cerebellar purkinje cells renders omega-agatoxin IVA-sensitive $\mathrm{Ca} 2+$ channels insensitive to inhibition by omega-conotoxin GVIA. J Pharmacol Exp Ther 324:806-814. CrossRef Medline

Urban NN, Henze DA, Barrionuevo G (2001) Revisiting the role of the hippocampal mossy fiber synapse. Hippocampus 11:408-417. CrossRef Medline

von Kitzing E, Jonas P, Sakmann B (1994) Quantal analysis of excitatory postsynaptic currents at the hippocampal mossy fiber-CA3 pyramidal cell synapse. Adv Second Messenger Phosphoprotein Res 29:235-260. CrossRef Medline

Vyleta NP, Jonas P (2014) Loose coupling between Ca2+ channels and release sensors at a plastic hippocampal synapse. Science 343:665-670. CrossRef Medline

Weber AM, Wong FK, Tufford AR, Schlichter LC, Matveev V, Stanley EF (2010) N-type Ca2 + channels carry the largest current: implications for nanodomains and transmitter release. Nat Neurosci 13:13481350. CrossRef Medline

Wheeler DB, Randall A, Tsien RW (1994) Roles of N-type and Q-type Ca2+ channels in supporting hippocampal synaptic transmission. Science 264: 107-111. CrossRef Medline

Wu LG, Saggau P (1995) Block of multiple presynaptic calcium channel types by omega-conotoxin-MVIIC at hippocampal CA3 to CA1 synapses. J Neurophysiol 73:1965-1972. Medline

Wu LG, Westenbroek RE, Borst JG, Catterall WA, Sakmann B (1999) Calcium channel types with distinct presynaptic localization couple differentially to transmitter release in single calyx-type synapses. J Neurosci 19: 726-736. Medline 